\title{
Probing ISM Structure in Trumpler 14 and Carina I Using the Stratospheric Terahertz Observatory 2
}

\author{
Young Min Seo ${ }^{1}$ (D), Paul F. Goldsmith ${ }^{1}$ (D), Christopher K. Walker ${ }^{2}$, David J. Hollenbach ${ }^{3}$, Mark G. Wolfire ${ }^{4}$, Craig A. Kulesa ${ }^{2}$, \\ Volker Tolls ${ }^{5}$, Pietro N. Bernasconi ${ }^{6}$, Ümit Kavak ${ }^{7,8}$, Floris F. S. van der Tak ${ }^{7,8}$, Russ Shipman ${ }^{8}$, Jian Rong Gao ${ }^{8,9}$, \\ Alexander Tielens ${ }^{10}$, Michael G. Burton ${ }^{11,12} \mathbb{D}$, Harold Yorke ${ }^{13}$, Erick Young ${ }^{13}$, William L. Peters ${ }^{2}$, Abram Young ${ }^{2}$, \\ Christopher Groppi ${ }^{14}$, Kristina Davis ${ }^{14,15}$, Jorge L. Pineda ${ }^{1}$ (D), William D. Langer ${ }^{1}$, Jonathan H. Kawamura ${ }^{1}$, Antony Stark ${ }^{5}$, \\ Gary Melnick $^{5}$, David Rebolledo ${ }^{16,17}$, Graeme F. Wong ${ }^{11,18}$, Shinji Horiuchi ${ }^{19}$, and Thomas B. Kuiper ${ }^{1}$ (D) \\ ${ }^{1}$ Jet Propulsion Laboratory, California Institute of Technology, 4800 Oak Grove Drive, Pasadena, CA 91109, USA; seo3919@gmail.com \\ ${ }^{2}$ Department of Astronomy \& Steward Observatory, University of Arizona, 933 North Cherry Avenue, Tucson, AZ 85721, USA \\ ${ }^{3}$ Carl Sagan Center, SETI Institute, 189 Bernado Avenue, Mountain View, CA 94043-5203, USA \\ ${ }^{4}$ Department of Astronomy, University of Maryland, College Park, MD 20742-2421, USA \\ ${ }^{5}$ Harvard-Smithsonian Center for Astrophysics, 60 Garden Street, Cambridge, MA 02138, USA \\ ${ }^{6}$ Applied Physics Laboratory, Johns Hopkins University, USA \\ ${ }^{7}$ Kapteyn Astronomical Institute, University of Groningen, The Netherlands \\ ${ }^{8}$ SRON Netherlands Institute for Space Research, Landleven 12, 9747 AD Groningen, The Netherlands \\ ${ }^{9}$ Faculty of Applied Science, Delft University of Technology, Delft, The Netherlands \\ ${ }^{10}$ Leiden Observatory, Leiden University, P.O. Box 9513, NL-2300 RA Leiden, The Netherlands \\ ${ }^{11}$ School of Physics, University of New South Wales, Sydney, NSW 2052, Australia \\ ${ }_{12}$ Armagh Observatory and Planetarium, College Hill, Armagh, BT61 9DG, UK \\ ${ }^{13}$ NASA Ames SOFIA Science Center, N211, Mountain View, CA 94043, USA \\ ${ }_{15}^{14}$ Department of Astronomy, Arizona State University, Tempe, AZ, USA \\ 15 University of California Santa Barbara, Santa Barbara, CA 93106, USA \\ ${ }^{16}$ Joint ALMA Observatory, Alonso de Córdova 3107, Vitacura, Santiago, Chile
${ }^{17}$ National Radio Astronomy Observatory, 520 Edgemont Road, Charlottesville, VA 22903, USA \\ ${ }^{18}$ School of Computing Engineering and Mathematics, Western Sydney University, Locked Bay 1797, Penrith, NSW 2751, Australia \\ ${ }^{19}$ CSIRO Astronomy and Space Science, Canberra Deep Space Communications Complex, P.O. Box 1035, Tuggeranong, ACT 2901, Australia \\ Received 2018 December 18; revised 2019 April 16; accepted 2019 May 7; published 2019 June 20
}

\begin{abstract}
We present observations of the Trumpler 14/Carina I region carried out using the Stratospheric Terahertz Observatory 2. The Trumpler 14/Carina I region is in the western part of the Carina Nebula Complex (CNC), which is one of the most extreme star-forming regions in the Milky Way. We observed Trumpler 14/Carina I in the $158 \mu \mathrm{m}$ transition of [C II] with a spatial resolution of $48^{\prime \prime}$ and a velocity resolution of $0.17 \mathrm{~km} \mathrm{~s}^{-1}$. The observations cover a $0^{\circ} .25$ by $0^{\circ} .28$ area with central position $l=297^{\circ} .34, b=-0^{\circ} .60$. The kinematics show that bright $[\mathrm{C} \mathrm{II}]$ structures are spatially and spectrally correlated with the surfaces of $\mathrm{CO}$ clouds, tracing the photodissociation region (PDR) and ionization front of each molecular cloud. Along seven lines of sight (LOSs) that traverse $\mathrm{Tr} 14$ into the dark ridge to the southwest, we find that the [C II] luminosity from the $\mathrm{H}$ II region is 3.7 times that from the PDR. In the same LOS, we find in the PDRs an average ratio of $1: 4.1: 5.6$ for the mass in atomic gas : dark CO gas : molecular gas traced by CO. Comparing multiple gas tracers, including $\mathrm{H} \mathrm{I} 21 \mathrm{~cm}$, [C II], CO, and radio recombination lines, we find that the $\mathrm{H}$ II regions of the $\mathrm{CNC}$ are well described as $\mathrm{H}$ II regions with one side freely expanding toward us, consistent with the Champagne model of ionized gas evolution. The dispersal of the GMC in this region is dominated by EUV photoevaporation; the dispersal timescale is 20-30 Myr.
\end{abstract}

Key words: ISM: kinematics and dynamics - ISM: structure - photon-dominated region (PDR) - stars: formation surveys - H II regions

\section{Introduction}

The interstellar medium (ISM) is one of the main constituents of galaxies, and understanding its life cycle has been a fundamental issue for following galaxy evolution, as well as star and planet formation. The ISM is observed to have multiple phases, including hot/warm ionized gas, warm/cold neutral gas, and cold molecular gas (see Snow \& McCall 2006, for a more detailed classification). The ISM cycles through these phases through dynamic processes including cloud formation, star formation, stellar winds, and supernova explosions. In the ISM life cycle, the transition from diffuse atomic gas to dense molecular clouds and the destruction of molecular clouds to diffuse gas by stellar feedback may be critical steps associated with star formation that may control the rate of star formation in galaxies. However, the ISM life cycle is still poorly understood because the transitions between the ISM phases go through multiple complex processes, and we lack high angular and spectral resolution surveys in the appropriate tracers to constrain transition mechanisms.

Emission of [C II] is closely related to the transition of the gas in the ISM between diffuse and dense phases. The [C II] $158 \mu \mathrm{m}$ line is one of the brightest and most widely distributed in the Milky Way, emitting up to $5 \%$ of the total far-IR in photodissociation regions (PDRs), and functions as a coolant for the cold neutral medium (e.g., Hollenbach \& Tielens 1997). Since its ionization energy $(11.6 \mathrm{eV})$ is lower than that of hydrogen, $\mathrm{C}^{+}$traces regions where $\mathrm{H}^{+}$is making the transition to $\mathrm{H}$ and $\mathrm{H}_{2}$. The [C II] emission is found in $\mathrm{HII}$ regions, $\mathrm{HI}$ 
regions, and $\mathrm{H}_{2}$ regions where the $\mathrm{CO}$ is photodissociated to $\mathrm{C}$ and $\mathrm{C}^{+}$(e.g., Pineda et al. 2013; Beuther et al. 2014; Langer et al. 2014; Velusamy et al. 2015; Pabst et al. 2017). Notably, [C II] is a tracer of the "CO-dark molecular gas" component of the ISM that cannot be seen in $\mathrm{H} \mathrm{I}$ or $\mathrm{CO}$ line emission. It can directly distinguish $\mathrm{H}$ I clouds from diffuse intercloud $\mathrm{H} \mathrm{I}$ gas, probe dense $\mathrm{H}_{2}$ gas not associated with $\mathrm{CO}$ emission (Langer et al. 2014), and trace mass flows from CO cloud surfaces (Orr et al. 2014).

The Carina Nebula Complex (CNC) is one of the most active star-forming regions in our galaxy. The CNC is roughly 400 times more luminous at optical wavelengths and 20 times larger in size than the Orion Nebula (Dias et al. 2002; O'Dell 2003, and reference therein). This makes the $\mathrm{CNC}$ a prominent laboratory for studying the life cycle of the ISM undergoing extreme star formation. The $\mathrm{CNC}$ is also frequently compared to 30 Doradus, which is an extreme star-forming region in the Large Magellanic Cloud. The CNC harbors many massive stars (at least 70 O-type and WR stars; Smith 2006) and has multiple phases of the ISM coexisting and transitioning from one to another as a result of the strong radiation from the massive stars. Many observations have been carried out in emission lines and continuum bands to probe the structures in the nebula (e.g., Zhang et al. 2001; Brooks et al. 2003; Oberst et al. 2011; Preibisch et al. 2012; Young et al. 2013; Hartigan et al. 2015; Rebolledo et al. 2016, 2017; Haikala et al. 2017). Integrated intensity maps in $\mathrm{H} \mathrm{I}, \mathrm{H} \alpha$, [O I], [C II], [C I], CO, and dust continuum have revealed $\mathrm{H}$ II regions, PDRs, globules, and dense clouds (Brooks et al. 2003; Oberst et al. 2011; Hartigan et al. 2015). High spectral resolution surveys in CO isotopologues and $\mathrm{H}$ I show a full complex of the diffuse and dense clouds in the nebula (Rebolledo et al. 2016, 2017). Harboring various phases and structures of the ISM interacting with star formation, the Carina Nebula is a unique test bed to study the transition of the ISM associated with massive star formation.

The ISM structure in the CNC has not been adequately probed due to a lack of high spatial and spectral resolution observations in tracers such as [C II], [N II], and [O I]. Oberst et al. (2011) carried out observations of [C II], [N II], and [O I] using the South Pole Imaging Fabry-Perot Interferometer and the Infrared Space Observatory (ISO). However, their study is limited to integrated intensity maps. The Mopra Southern Galactic Plane $\mathrm{CO}$ survey observed the $\mathrm{CNC}$ with a high spectral resolution of $0.088 \mathrm{~km} \mathrm{~s}^{-1}$ and revealed that molecular clouds have highly complex structures (Rebolledo et al. 2016). The CO spectral maps indicate that not only are there many molecular clouds and globules distributed throughout this region, but there are also multiple velocity components along certain lines of sight (LOSs) within molecular clouds (e.g., Carina I and II). The complexity of the structure of the molecular gas indicates that the ISM structures in the CNC must be probed using observations with high spatial and spectral resolution.

In this study, we report a high spatial and spectral resolution survey toward the Trumpler 14 and Carina I ( $\operatorname{Tr} 14 /$ Carina I) region in the [C II] $158 \mu \mathrm{m}$ transition using the Stratospheric Terahertz Observatory 2 (STO2). An open cluster, $\operatorname{Tr} 14$ is located half a degree $(20 \mathrm{pc})$ west of the blue variable star $\eta$ Carinae, and Carina $I$ is a dense cloud forming a dust lane located to the south of $\operatorname{Tr} 14$ and illuminated by both $\operatorname{Tr} 16$ (located $30^{\prime}$ to the east of $\operatorname{Tr} 14$ and containing $\eta$ Carinae) and $\operatorname{Tr} 14$. The $\operatorname{Tr} 14 /$ Carina I region contains multiple phases of the ISM in H II regions, PDRs, and dense molecular clouds. The key output of our survey is a high spatial and spectral resolution data cube in the [C II] $158 \mu \mathrm{m}$ transition toward the Carina Nebula. Here we use our [C II] map to study the physical structures of the ISM, including PDRs, molecular clouds, and $\mathrm{H}$ II regions in the CNC.

This study contains extensive analysis with many new findings. The following are the highlights of this study and can be found in the discussion and conclusions sections. Comparing our [C II] spectral map to the Mopra CO map, we found that bright [C II] emission in the $\mathrm{CNC}$ is closely related to the $\mathrm{CO}$ clumps in position-position-velocity (PPV) space, suggesting that bright [C II] emission likely arises from PDR and ionization fronts. We also found large absorption cavities in H I $21 \mathrm{~cm}$ emission and that those cavities are in good agreement with the $\mathrm{CO}$ clouds/clumps and the Keyhole Nebula, a CO-dark molecular cloud, in positionposition space. On the other hand, the velocities of the absorption cavities are $\pm 5-10 \mathrm{~km} \mathrm{~s}^{-1}$ shifted from the $\mathrm{CO}$ velocity centroids, suggesting that the cavities may follow cold $\mathrm{H}$ I gas photoevaporating or stripped from cloud surfaces. Through detailed PDR modeling of 10 different regions representing various ISM structures, we found a mass proportion of 1:4.1:5.6 for the atomic:dark:molecular(CO) gas and that six out of 10 regions are dominated by [C II] emission from $\mathrm{H}$ II regions rather than PDRs. Finally, combining kinematics and modelings, we found that the three-dimensional morphology of the $\mathrm{CNC}$ is consistent with one side of numerous blister $\mathrm{H}$ II regions expanding freely toward us, similar to a Champagne flow, with a lifetime of $\mathrm{CO}$ clouds exposed to $\mathrm{H}$ II regions being 20-30 Myr.

We describe details of observations using STO2 and data reduction in Section 2. We show results and analysis along with complementary observations, including dust continuum, $\mathrm{CO}, \mathrm{H} 92 \alpha$, H I $21 \mathrm{~cm}$, and Gaia Sky survey, in Sections 3 and 4. In Section 5, we present detailed modeling of PDRs and its implications for the ISM structures in the $\operatorname{Tr} 14 /$ Carina I region. In Section 6, we discuss a possible three-dimensional morphology of the Tr 14/Carina I region and uncertainties of data. We also discuss photoevaporation and mass loss of giant molecular clouds (GMCs) by EUV. Finally, in Section 7 we summarize our results.

\section{Observation and Data Reduction}

The STO2 is a balloon-borne observatory designed to fly in the stratosphere at $38 \mathrm{~km}$ altitude to avoid the severe atmospheric absorption at submillimeter wavelengths from ground-based sites. It consists of a $0.8 \mathrm{~m}$ telescope, a terahertz heterodyne receiver, and a high-resolution fast Fourier transform spectrometer $(1 \mathrm{MHz})$ with 1024 channels (Table 1). The STO2 was launched on 2016 December 7 and flew until 2016 December 29 over the Antarctic continent and surveyed galactic plane and star-forming regions including the CNC. The observations were made in two modes: on-the-fly (OTF) mapping and spiral mapping. The OTF observations were done with a typical spacing of a half beam size between raster observation lines and a relatively short integration time $(0.65 \mathrm{~s})$ per OTF dump, while the spiral observations were made with a sparse pointing ( $>2$ FWHM beam size) and longer integration times $(>1 \mathrm{~s})$. The maximum observation duration per raster line is set to be smaller than $35 \mathrm{~s}$, which is the typical Allan variance time of the STO2 receivers. The telescope pointing was controlled by an onboard star tracker, and the typical pointing accuracy during the OTF mode was measured to be less than $15^{\prime \prime}$. For more details about the STO2 instrument and mission, see C. K. Walker et al. (2019, in preparation). 
Observations of the CNC were centered on a position near the center of the $\operatorname{Tr} 14$ cluster at $(l, b)=+287.33,-0.601$ covering 0.25 by $0^{\circ} .28$ in Galactic coordinates. The observations were done in the OTF mode. One OTF scan contains 45-47 spectra ( $\sim 12^{\prime \prime}$ spacing) observing a 0.14 strip in Galactic latitude, which is half the map size. The native beam size at $158 \mu \mathrm{m}$ is $48^{\prime \prime}(0.53 \mathrm{pc}$ at a distance of $2.3 \mathrm{kpc})$. The distance to the CNC is still debated and reported between 2.2 and $2.8 \mathrm{kpc}$; see Astraatmadja \& Bailer-Jones (2016), Gaia Collaboration et al. (2016a, 2016b), Lindegren et al. (2016), Smith (2006), Smith \& Brooks (2008), and Smith \& Stassun (2017) for more details. Spectra cover a local sidereal time velocity range from -112 to $57 \mathrm{~km} \mathrm{~s}^{-1}$ with a spectral resolution of $1 \mathrm{MHz}\left(0.17 \mathrm{~km} \mathrm{~s}^{-1}\right.$ at $\left.1.9 \mathrm{THz}\right)$. For subtraction of broadband emission, observations toward a nearby reference position were made at the beginning and end of each OTF scan. We selected a reference position $(l, b=+286.50,+0.200)$ based on the lowest $[\mathrm{C} \mathrm{I}]{ }^{3} P_{1}-{ }^{3} P_{0} / \mathrm{CO} J=4-3$ intensity ratio, indicative of minimal [C II] emission (Zhang et al. 2001). The spectra toward the reference position are typically of good quality but vary slowly in time. To obtain an accurate reference spectrum for a given OTF scan, we linearly interpolated the reference spectra in time. The single sideband system temperature was $3300 \mathrm{~K}$ with a typical variation of $100 \mathrm{~K}$ at 1.9 THz during the CNC observations.

The STO2 data were reduced using the STO2 pipeline (Y. M. Seo et al. 2019, in preparation). The STO2 pipeline is designed to process spectral scans considering unique characteristics found in the STO2 data. For example, some of the STO2 spectra have large fringes $(>50 \mathrm{~K})$ with their patterns varying over short periods $(<60 \mathrm{~s})$. We could not effectively defringe the data with conventional observation software (e.g., CLASS). We wrote the STO2 pipeline to suppress large fringes by interpolating reference scans and using machine-learning algorithms. The machine-learning algorithms characterized large-amplitude fringe patterns (using, e.g., deflation-independent component analysis; Hyvärinen \& Oja 2000) and identified extremely noisy spectra (using clustering algorithms on spectrum properties). For the CNC observations, $90 \%$ of the spectra were of sufficient quality to be included in the final spectral map. In the reduced spectra, the typical overall noise level is $1.3 \mathrm{~K}$ in main beam temperature, which is slightly larger than the expected radiometric noise $(0.8 \mathrm{~K})$ due to the residual effects of fringe. The regridding of spectra was done following algorithms shown in Mangum et al. (2000) using a Gaussian-Bessel kernel without any weighting but omitting exceptionally noisy spectra. The final effective beam size in the spectral map is $55^{\prime \prime}$.

The intensities of the STO2 observations toward the CNC were calibrated using ISO [C II] data (Oberst et al. 2011). There were 12 positions observed by both STO2 and ISO. From a comparison of observed intensities toward these positions, we estimate the main beam efficiency of STO2 to be $0.7_{-0.08}^{+0.14}$. We therefore adopt a main beam efficiency of 0.7 in the analysis of STO2 data (for more detail, see Appendix A).

\section{Integrated Intensity of [C II] Emission}

\subsection{Spatial Distribution of Integrated [C II] Emission}

We present the integrated intensity of the [C II] emission in Figures 1 and 2 together with the $\mathrm{H} \alpha$ image from Hubble (Smith 2006), the $8 \mu \mathrm{m}$ image from Spitzer (Smith et al. 2010;
Povich et al. 2011), the 160 and $500 \mu \mathrm{m}$ images from Herschel (Preibisch et al. 2012; Gaczkowski et al. 2013; Roccatagliata et al. 2013), the integrated H I $21 \mathrm{~cm}$ image from ATCA (Rebolledo et al. 2017), the integrated CO 1-0 emission from Mopra (Rebolledo et al. 2016), and the integrated $\mathrm{H} 92 \alpha$ emission from the Deep Space Network (DSN). Using multiple continuum and spectral line images, we describe here structures of the $\operatorname{Tr} 14 /$ Carina I region and investigate the spatial distribution of the [C II] emission with respect to different ISM phases.

The overall morphology of the $\operatorname{Tr} 14$ and $\operatorname{Tr} 16$ region is as follows: the $\operatorname{Tr} 14 /$ Carina I region is located in the western part of the $\mathrm{CNC}$, while the $\operatorname{Tr} 16$ region is located in the eastern part. The Tr 14 cluster is partially surrounded by dense clouds, including the dark $\mathrm{V}$-shaped dust lane (see $\mathrm{H} \alpha$ in Figure 1) in the south of $\operatorname{Tr} 14$ (aka Carina I) and dense CO clouds to the west and north of $\operatorname{Tr} 14$ (see ${ }^{13} \mathrm{CO} 1-0$ contours in Figure 2). The east side of the $\operatorname{Tr} 14 /$ Carina I region is open to the $\eta$ Carinae and $\operatorname{Tr} 16$ region, but there is another dust lane to the east of $\eta$ Carinae, suggesting that the dust lane and dense clouds partially surround the $\eta$ Carinae and $\operatorname{Tr} 14$ region. The optical image shows that a majority of the members of the $\mathrm{Tr}$ 14 and Tr 16 clusters have low extinction (e.g., Smith 2006), which indicates that there is no significant foreground cold gas toward the two clusters and the H II regions are exposed to us. On the other hand, the V-shaped dust lane appears as a highextinction region in the $\mathrm{H} \alpha$ image, suggesting that the dust lane is in front of the H II region (e.g., Wu et al. 2018).

We find that the [C II] emission covers a significant fraction of the area mapped using STO2 $(0.25 \times 0.28$, equivalent to $10.0 \mathrm{pc} \times 11.2 \mathrm{pc}$ at a distance of $2.3 \mathrm{kpc}$ ). The fraction of the area with peak main beam temperatures $>5$ and $>10 \mathrm{~K}$ are $83 \%$ and $58 \%$, respectively. The [C II] emission is expected from both the $\mathrm{H}$ II region of $\operatorname{Tr} 14$ and the PDRs. Looking at the $\mathrm{H} \alpha$ and $8 \mu \mathrm{m}$ maps, we find that the upper half of our [C II] map coincides with the $\mathrm{H}$ II region of $\operatorname{Tr} 14$, and the other half of the [C II] map is coincident with the Carina I cloud and its PDRs, which confirms that there are multiple sources for the [C II] emission. The brightest intensity peak of the [C II] emission is $370 \mathrm{~K} \mathrm{~km} \mathrm{~s}^{-1}$, located $7^{\prime}$ south of $\operatorname{Tr} 14(4.7 \mathrm{pc}$ at the distance of $2.3 \mathrm{kpc}$ ), where the Carina I-E/Carina I-W clouds are located (indicated by green arrows in Figure 1). The brightest emission at Carina I-E/Carina I-W is because they are the densest clouds in Carina I and irradiated by a B1 supergiant only 0.5 pc away in projected distance, in addition to the main members of the $\operatorname{Tr} 14$ cluster, thus resulting in a significantly high emission measure (the excitation condition of these clouds is further discussed in Section 5).

We compare the integrated [C II] emission to the dust continuum emission and PAH observed using Herschel and Spitzer. The dust continuum emission at 160 and $500 \mu \mathrm{m}$ shows warm and cold dust structures in the $\operatorname{Tr} 14 /$ Carina I region. The $8 \mu \mathrm{m}$ emission from Spitzer is typically dominated by $\mathrm{PAH}$ emission, which traces PDRs in star-forming regions. Overall, the strong [C II] emission agrees better on a large scale with the bright structures seen in dust and PAH emission than with the bright structure of the $\mathrm{H} \alpha$ emission, suggesting that the strong [C II] emission may originate from PDR and $\mathrm{H}$ II regions near ionization fronts, while we still observe weak [C II] emission coming from the inner part of the $\operatorname{Tr} 14 \mathrm{H}$ II region.

We show the integrated ${ }^{12} \mathrm{CO}$ and ${ }^{13} \mathrm{CO} 1-0$ observed using Mopra in Figures 1 and 2 (Rebolledo et al. 2016), along with the integrated $[\mathrm{C} \mathrm{II}]$ emission. The ${ }^{12} \mathrm{CO} 1-0$ map reveals the 


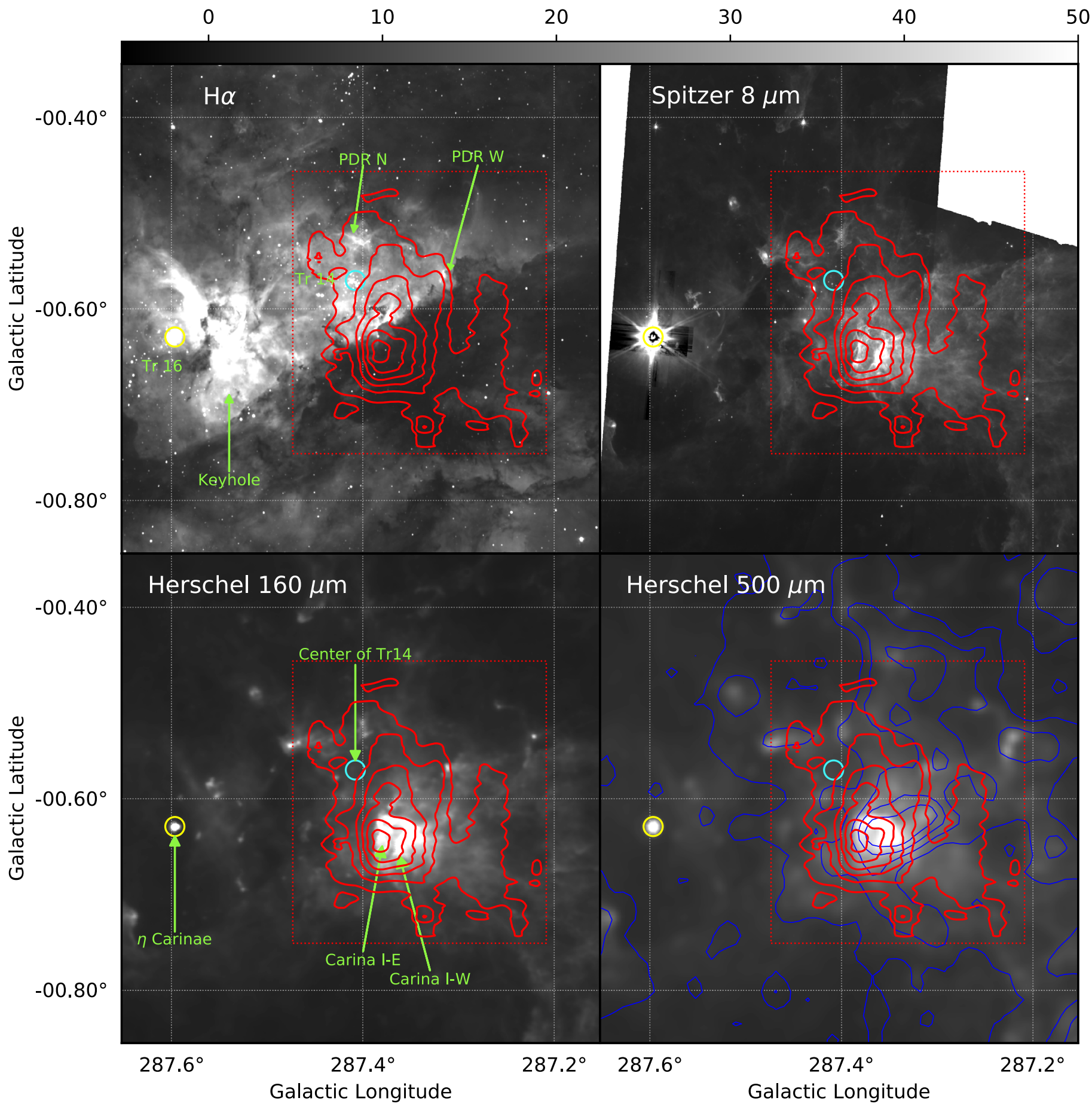

Figure 1. Integrated intensity of [C II] $158 \mu \mathrm{m}$ (red contours) overlaid on $\mathrm{H} \alpha$ and 8, 160, and $500 \mu \mathrm{m}$ continuum images. The images are displayed in Galactic coordinates. The [C II] intensity contours are at $100,150,200,250,300$, and $350 \mathrm{~K} \mathrm{~km} \mathrm{~s}^{-1}$. The [C II] emission is integrated from -40 to $0 \mathrm{~km} \mathrm{~s}{ }^{-1}$. The box defined by the red dotted lines indicates the area mapped in [C II]. The blue contours in the bottom right panel are the integrated intensity of ${ }^{12} \mathrm{CO} 1-0$ at levels of $20,60,100$, 140 , and $180 \mathrm{~K} \mathrm{~km} \mathrm{~s}^{-1}$. The ${ }^{12} \mathrm{CO} 1-0$ emission is integrated from -40 to $0 \mathrm{~km} \mathrm{~s}^{-1}$. The gray-scale bar gives the intensity of the continuum images; the units for the $\mathrm{H} \alpha$ and 8,160, and $500 \mu \mathrm{m}$ images are 400 counts (see Smith 2006) and 200, 400, and $200 \mathrm{MJy} \mathrm{s}^{-1}$, respectively. The yellow and cyan circles denote $\eta$ Carinae, which is a member of $\operatorname{Tr} 16$, and the center of $\operatorname{Tr} 14$. The $\operatorname{Tr} 14$ and $\operatorname{Tr} 16$ clusters are also indicated in the first panel. The bright PDRs, the Keyhole Nebula, Carina $\mathrm{I}-\mathrm{E}$, and Carina I-W are indicated by the green arrows.

cold molecular ISM, and the ${ }^{13} \mathrm{CO} 1-0$ map highlights the denser portions of the CO clouds in the Tr 14/Carina I region. The overall spatial distribution of the CO 1-0 emission, particularly ${ }^{13} \mathrm{CO} 1-0$, shows that $\mathrm{CO}$ clouds form a wall bounding the western part of the $\operatorname{Tr} 14 /$ Carina I region. There is also weak, broad ${ }^{12} \mathrm{CO} 1-0$ emission from $\operatorname{Tr} 14$, suggesting that there may be $\mathrm{CO}$ gas behind $\operatorname{Tr} 14$, since we do not see significant extinction in optical bands. We find that, overall, the [C II] emission is broadly distributed covering $\operatorname{Tr} 14$ and nearby CO clumps, while the $\mathrm{CO}$ emission is bright $4^{\prime}$ west and $5^{\prime}$ south of $\operatorname{Tr} 14$ (2.6 and $3.4 \mathrm{pc}$ at a distance of $2.3 \mathrm{kpc}$ ) and extended to the western part of the $\operatorname{Tr} 14 /$ Carina I region. There is a region with relatively bright [C II] emission $\left(121 \mathrm{~K} \mathrm{~km} \mathrm{~s}^{-1}\right)$ but quite weak $\mathrm{CO}$ emission (integrated 


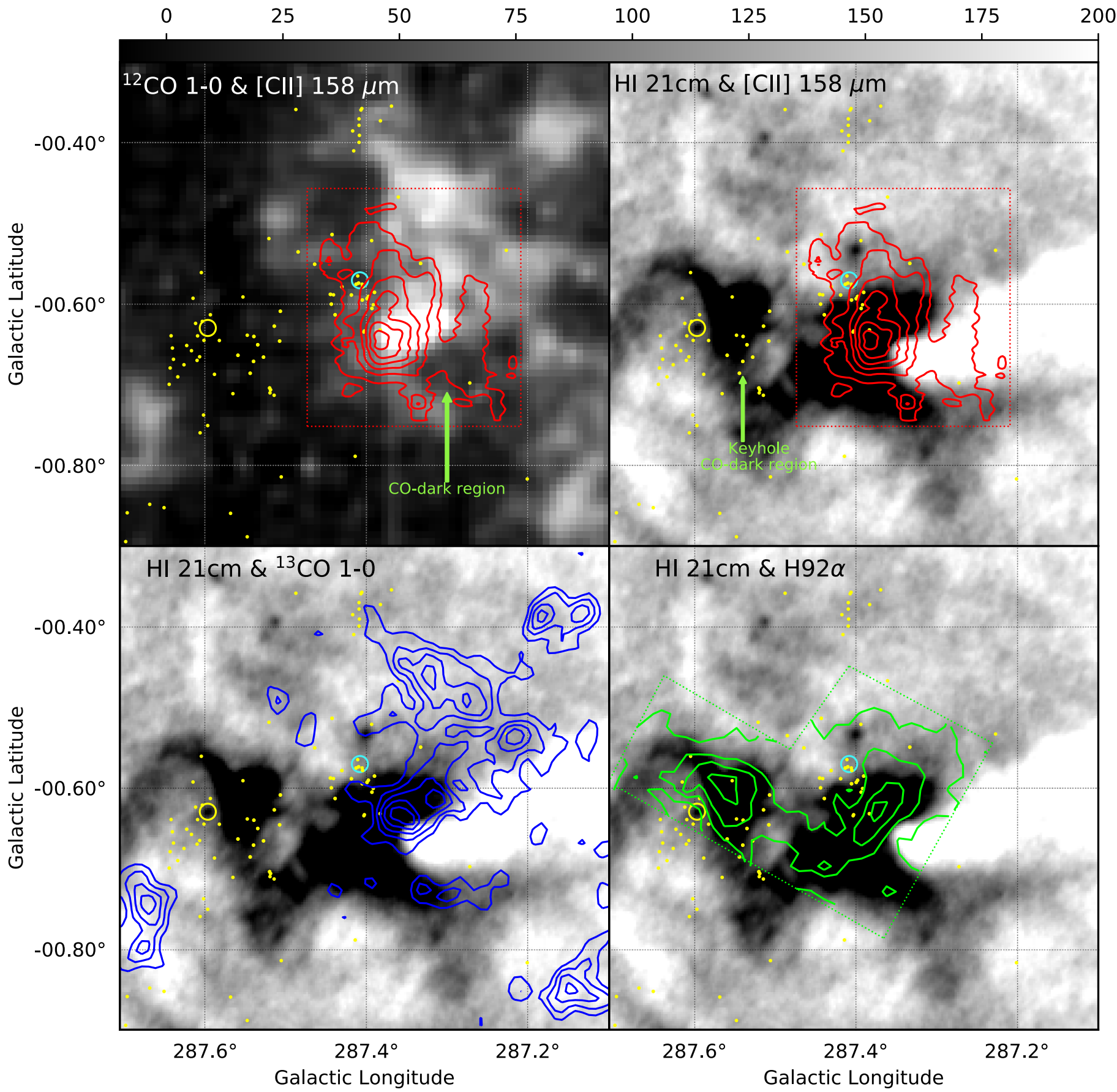

Figure 2. Integrated intensity of [C II] $158 \mu \mathrm{m}$ (red contours) overlaid on the integrated ${ }^{12} \mathrm{CO}$ and $\mathrm{H} \mathrm{I} 21 \mathrm{~cm}$ images. The [C II], ${ }^{12} \mathrm{CO}$, and $\mathrm{HI} 21 \mathrm{~cm}$ emission is integrated from -40 to $0 \mathrm{~km} \mathrm{~s}^{-1}$. The images are displayed in Galactic coordinates. The [C II] intensity contours are at 100, 150, 200, 250, 300, and 350 K km s The $\left[\mathrm{C} \mathrm{II]} \mathrm{emission} \mathrm{is} \mathrm{integrated} \mathrm{from}-40\right.$ to $0 \mathrm{~km} \mathrm{~s}^{-1}$. The integrated intensity of ${ }^{13} \mathrm{CO} 1-0$ (blue) and $\mathrm{H} 92 \alpha$ (green) emission is overlaid on the $\mathrm{H} \mathrm{I} 21 \mathrm{~cm}$ emission in the third and fourth panels. The ${ }^{13} \mathrm{CO} 1-0$ contours are at $8,12,16,20$, and $24 \mathrm{~K} \mathrm{~km} \mathrm{~s}^{-1}$, and the $\mathrm{H} 92 \alpha$ contours are at $20,40,60$, and $80 \mathrm{~K} \mathrm{~km} \mathrm{~s}{ }^{-1}$. The ${ }^{13} \mathrm{CO} 1-0$ emission is integrated from -40 to $0 \mathrm{~km} \mathrm{~s}^{-1}$, and the $\mathrm{H} 92 \alpha$ emission is integrated from -65 to $20 \mathrm{~km} \mathrm{~s}^{-1}$. The red and green dotted boxes define the area of the [C II] and $\mathrm{H} 92 \alpha$ observations, respectively. The gray-scale bar shows the scale of the integrated ${ }^{12} \mathrm{CO}$ and $\mathrm{H} \mathrm{I} 21 \mathrm{~cm}$ images. The scale values are to be multiplied by 0.8 and $10 \mathrm{~K} \mathrm{~km} \mathrm{~s}^{-1}$ for the integrated ${ }^{12} \mathrm{CO}$ and $\mathrm{H} \mathrm{I} 21 \mathrm{~cm}$ images, respectively. The yellow and cyan circles denote $\eta$ Carinae and the center of Tr 14 . The yellow dots denote O- and B-type stars (Alexander et al. 2016). The CO-dark region in the Tr 14/Carina I region and the Keyhole Nebula, which is another CO-dark cloud, are indicated by the green arrows.

intensity $34 \mathrm{~K} \mathrm{~km} \mathrm{~s}^{-1}$ compared to $211 \mathrm{~K} \mathrm{~km} \mathrm{~s}^{-1}$ from Carina $\mathrm{I}-\mathrm{E})$, which may indicate a CO-dark molecular region (Langer et al. 2014; indicated by a green arrow in the first panel of Figure 2). The intensity peaks of individual CO clumps are typically displaced a couple of arcminutes relative to the [C II] and PAH $(8 \mu \mathrm{m})$ intensity peaks, showing the locations of $\mathrm{CO}$ clumps relative to their PDRs and ionization fronts.
We probe the spatial distribution of the cold neutral medium using the integrated $\mathrm{HI}$ emission and the [C II] emission (Figure 2). We integrate the $\mathrm{H} \mathrm{I}$ emission from -40 to $0 \mathrm{~km} \mathrm{~s}^{-1}$ because most of the $\mathrm{CO}$ and $[\mathrm{CII}]$ emission is within this velocity range. The integrated $\mathrm{HI} 21 \mathrm{~cm}$ emission shows extended HI emission covering the CNC with clumpy $\mathrm{HI}$ clouds and with cavities in the $\mathrm{H}$ I emission near Tr 14 and $\eta$ 
Carinae. Cavities may appear when neutral atomic hydrogen forms molecular hydrogen or becomes ionized, or if there is foreground absorption due to cooler H I gas. The cavities in the $\mathrm{CNC}$ are due to the absorption features in the H I spectra. We also find that the spatial distribution of the cavities agrees well with locations of the Keyhole Nebula and the dense CO clouds near $\operatorname{Tr} 14$. To have absorption features, there must be a continuum background. In the $\mathrm{CNC}$, the free-free emission of the $\mathrm{H}$ II region provides a bright, hot background against which we see absorption. We find that the distribution of the $\mathrm{H} 92 \alpha$ emission is broadly extended, including the $\mathrm{H}$ II region and the cavities. Comparing H I $21 \mathrm{~cm}$ to $[\mathrm{C} \mathrm{II}]$ and ${ }^{12} \mathrm{CO} 1-0$, we find numerous bright $\mathrm{CO}$ clumps and $[\mathrm{C}$ II] emission within the cavities in the $\operatorname{Tr} 14 /$ Carina I region. In this part of the cavity, the cold $\mathrm{HI}$ in dense $\mathrm{CO}$ clumps may be the source of absorption, which was also discussed in Rebolledo et al. (2017). On the other hand, we do not see significant CO emission in the cavity west of $\eta$ Carinae, where the Keyhole Nebula is located. This suggests that the Keyhole Nebula is a CO-dark cloud with relatively cold H I together with H II gas.

\section{Kinematics of $[\mathrm{C} \mathrm{II}]$ and Other Tracers in the $\operatorname{Tr} 14 /$ Carina I Region}

Here we discuss the structure and kinematics of ionized, neutral, and molecular gas in the Tr 14/Carina I region in PPV space. We analyze the channel maps and spectra of our [C II] $158 \mu \mathrm{m}$ observation along with other observations, including $\mathrm{H} 92 \alpha,{ }^{12} \mathrm{CO}$ and ${ }^{13} \mathrm{CO} 1-0$ (Rebolledo et al. 2016), $\mathrm{H} \alpha$ (Smith 2006), and HI $21 \mathrm{~cm}$ (Rebolledo et al. 2017). To disentangle the complicated ISM structure in the CNC, we first focus on the dense cloud/clumps and their PDRs traced by $\mathrm{CO}$ and $[\mathrm{CII}]$ and expand our view to the ionized and neutral atomic media traced by optical lines (H $\alpha,[\mathrm{N} \mathrm{II}] 6548 \AA$; Damiani et al. 2016), radio recombination lines (H92 $\alpha$ ), and H I $21 \mathrm{~cm}$ (Rebolledo et al. 2017).

\subsection{Channel Maps of [C II] $158 \mu \mathrm{m}$ and CO 1-O}

Figure 3 shows the channel maps of [C II] $158 \mu \mathrm{m}$ and ${ }^{12} \mathrm{CO}$ 1-0 (Rebolledo et al. 2016) overlaid on $\mathrm{H} \alpha$ (Smith 2006). The [C II] emission is mostly found in the velocity range from -32 to $-5 \mathrm{~km} \mathrm{~s}^{-1}$ and is spatially localized near $\operatorname{Tr} 14$. We find that the ${ }^{12} \mathrm{CO} 1-0$ emission also covers the velocity range from -32 to $-5 \mathrm{~km} \mathrm{~s}^{-1}$ but spans a wider area west of $\operatorname{Tr} 14$ compared to the $[\mathrm{C} \mathrm{II}]$ emission. We find no significant $\mathrm{CO}$ emission between $\eta$ Carinae and $\operatorname{Tr} 14$ in any channel map. We find weak $\mathrm{CO}$ emission east of $\eta$ Carinae but at slightly blueshifted velocity $\left(-25 \mathrm{~km} \mathrm{~s}^{-1}\right)$ compared the $\mathrm{CO}$ emission west of $\operatorname{Tr} 14\left(-20 \mathrm{~km} \mathrm{~s}^{-1}\right)$.

Based on the distribution of the [C II] $158 \mu \mathrm{m}$ and ${ }^{12} \mathrm{CO} 1-0$ emission in PPV space, we may divide the dense structures into three velocity groups. The first group includes the ${ }^{12} \mathrm{CO}$ and [C II] structures in the local standard of rest (LSR) velocity range from -32 to $-23 \mathrm{~km} \mathrm{~s}^{-1}$, the second group includes the dense structures in the velocity range from -23 to $-13 \mathrm{~km} \mathrm{~s}^{-1}$, and the third group includes the dense structures in the LSR velocity range from -13 to $-5 \mathrm{~km} \mathrm{~s}^{-1}$.

The first group ( -32 to $-23 \mathrm{~km} \mathrm{~s}^{-1}$ ) contains a few $\mathrm{CO}$ clumps south of $\operatorname{Tr} 14$, including a part of Carina I-E/Carina $\mathrm{I}-\mathrm{W}$ and the $\mathrm{CO}$ clumps east of $\eta$ Carinae. This group is likely in front of $\operatorname{Tr} 14$ relative to us, since CO clumps in this velocity range appear as dark clumps with respect to the bright $\mathrm{H} \alpha$ background (Haikala et al. 2017). Also, their blueshifted velocity compared to the LSR velocity of most of the CO clumps suggests that they are pushed toward to us by expanding $\mathrm{H}$ II gas and in the foreground of the H II region. The CO clumps in this group are relatively isolated from each other and have bright [C II] layers on their outskirts (for more detail, see the maps at -28.5 and $-23.5 \mathrm{~km} \mathrm{~s}^{-1}$ in Appendix B), which indicates the presence of ionization fronts and PDRs surrounding those CO clumps due to high-mass stars in the Tr 14/Carina I region. We find that the $[\mathrm{C} \mathrm{II}]$ emission is mostly located in the eastern outskirts of the $\mathrm{CO}$ clumps rather than in the northern outskirts facing the center of $\operatorname{Tr} 14$. This may be due to the B1 supergiant and $\mathrm{O} 7$ binary on the east side of Carina I (Wu et al. 2018). The spatial distribution of $\mathrm{CO}$ and $[\mathrm{C}$ II] emission suggests that the CO clumps in the first group may not be at the same distance from us as $\operatorname{Tr} 14$, which is consistent with silhouette globules at velocity ranges from -30 to $-20 \mathrm{~km} \mathrm{~s}^{-1}$ facing toward $\operatorname{Tr} 16$ rather than Tr 14 (Smith et al. 2003).

Near $\eta$ Carinae, we see that there are CO clumps to the east at $-28.5 \mathrm{~km} \mathrm{~s}^{-1}$, which comprises the east dust lane of the CNC. These CO clumps are blueshifted compared to the $\mathrm{CO}$ cloud to the west, and their LSR velocity is similar to that of the CO clumps in the dust lane south of $\operatorname{Tr} 14$. This suggests that CO clumps to the east of $\eta$ Carinae are likely in the foreground of the high-mass stars in the $\mathrm{CNC}$. We do not see any strong $\mathrm{CO}$ emission near $\eta$ Carinae in any channels, while there are CO clumps near $\operatorname{Tr} 14$ in the redshifted velocity range of -16 to $-8 \mathrm{~km} \mathrm{~s}^{-1}$. It is likely that $\eta$ Carinae may have cleared out the dense structures where it originally formed, while $\operatorname{Tr} 14$ is still interacting with nearby dense clumps. This picture is consistent with the younger age of $\operatorname{Tr} 14$ compared to Tr 16 (Walborn 1973; Morrell et al. 1988; Vazquez et al. 1996; Smith \& Brooks 2008; Rochau et al. 2011), suggesting that $\operatorname{Tr} 14$ has not lived long enough to clear out its surroundings. We will further discuss the three-dimensional structure of the $\mathrm{Tr}$ 14/Carina I region in Section 6.

The second group $\left(-23\right.$ to $\left.-15 \mathrm{~km} \mathrm{~s}^{-1}\right)$ includes the $\mathrm{CO}$ clouds/clumps west of $\operatorname{Tr} 14$. In this velocity range, we find that the $\mathrm{CO}$ emission is brighter and more extended than the [C II] emission in the other groups, suggesting that the majority of the $\mathrm{CO}$ gas is within this velocity range. The spatial distribution shows highly clumpy $\mathrm{CO}$ structures but also includes a dense $\mathrm{CO}$ "wall" to the west of $\operatorname{Tr} 14$ in the LSR velocity range from -20 to $-15 \mathrm{~km} \mathrm{~s}^{-1}$ with their central velocity near $-17 \mathrm{~km} \mathrm{~s}^{-1}$. The [C II] emission reveals isolated structures associated with the CO clumps in the LSR velocity range from -23 to $-20 \mathrm{~km} \mathrm{~s}^{-1}$. The [C II] emission associated with the CO clump is likely due to the PDRs of the clump. On the other hand, at velocities from -20 to $-15 \mathrm{~km} \mathrm{~s}^{-1}$, we find that the [C II] emission forms a thick strip following the eastern outskirts of the $\mathrm{CO}$ wall. The bright [C II] strip is the ionization front of the dense $\mathrm{CO}$ wall.

In the third group $\left(-15\right.$ to $\left.-5 \mathrm{~km} \mathrm{~s}^{-1}\right)$, the ${ }^{12} \mathrm{CO}$ emission is found on and around the center of $\operatorname{Tr} 14$. Considering that the extinction from $\mathrm{H} \alpha$ is quite low in this direction (Smith 2006; Hur et al. 2015), we think that the $\mathrm{CO}$ gas in this group is likely behind Tr 14 along our LOS. In the [C II] channel maps, we find that the majority of the [C II] emission is found to be spatially associated with CO clumps (e.g., see channel map at $-11 \mathrm{kms}^{-1}$ in Appendix B). This indicates that the [C II] emission in this group comes from the PDRs of the CO clumps, which are behind $\operatorname{Tr} 14$ and may be being pushed away from us.

Beyond $-5 \mathrm{~km} \mathrm{~s}^{-1}$, there is weak ${ }^{12} \mathrm{CO} 1-0$ emission around $\operatorname{Tr} 14$, but we could not find a significant dense cloud. This 


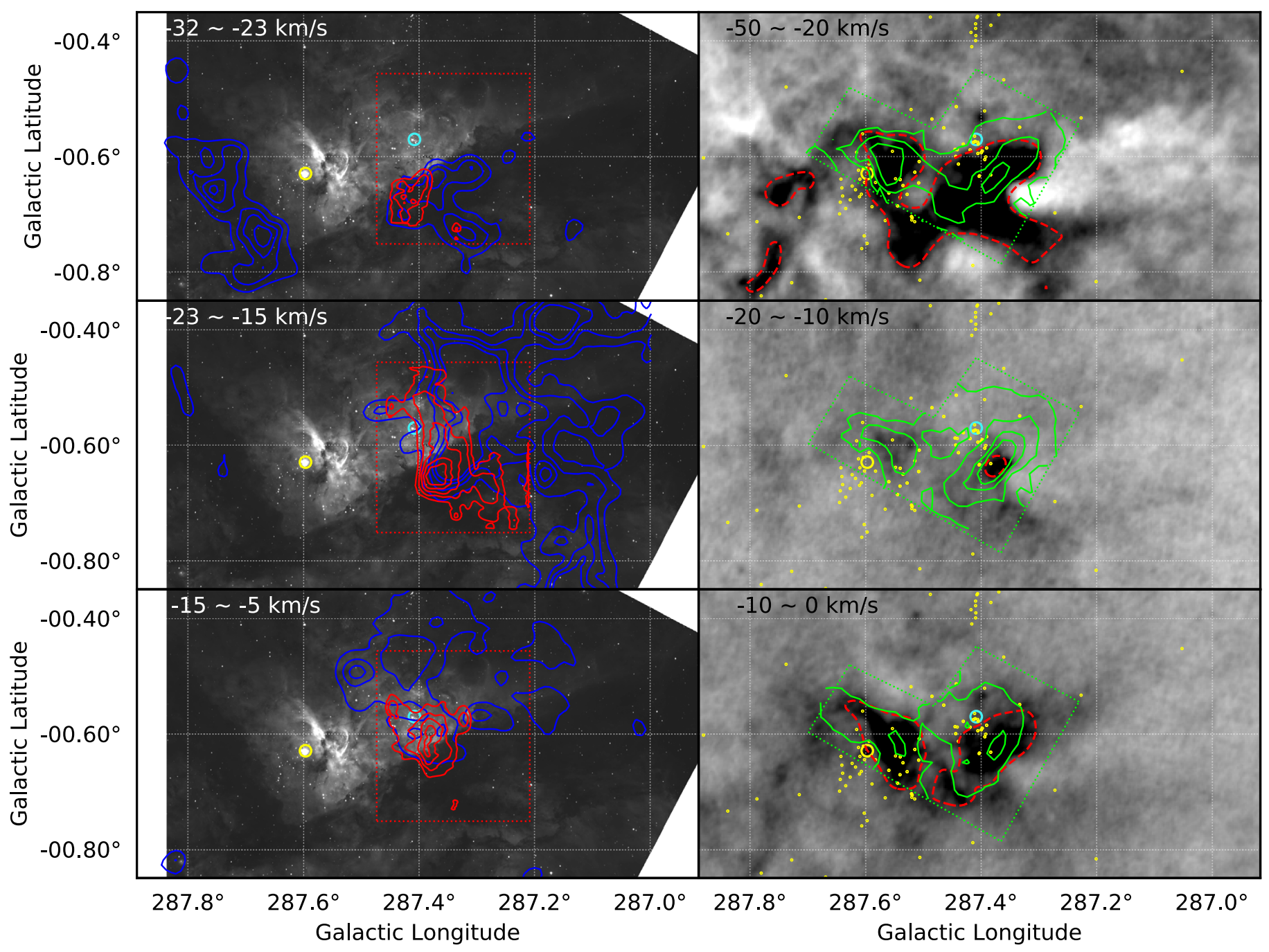

Figure 3. Channel maps of [C II] $158 \mu$ m obtained with STO2 (red contours), ${ }^{12} \mathrm{CO}$ observed with Mopra (blue contours; Rebolledo et al. 2016), H92 $\alpha$ (green contours), and H I $21 \mathrm{~cm}$ (gray-scale background in the right column; Rebolledo et al. 2017). The gray-scale background in the left column is an H $\alpha$ image (Smith 2006). The $[\mathrm{C} \mathrm{II}]$ contours start at $40 \mathrm{~K} \mathrm{~km} \mathrm{~s}^{-1}$ and increase in $20 \mathrm{~K} \mathrm{~km} \mathrm{~s}^{-1}$ increments. The ${ }^{12} \mathrm{CO}$ contours start at $10 \mathrm{~K} \mathrm{~km} \mathrm{~s}$ and increase in $10 \mathrm{~K} \mathrm{~km} \mathrm{~s}$ increments. The $\mathrm{H} 92 \alpha$ contours are at $10,20,30$, and $40 \mathrm{~K} \mathrm{~km} \mathrm{~s}^{-1}$ in the top panel and 5, 10, 15, and $20 \mathrm{~K} \mathrm{~km} \mathrm{~s}^{-1}$ in the middle and bottom panels. The red and green boxes with the dotted lines are the areas mapped in [C II] and $\mathrm{H} 92 \alpha$, respectively. The red dashed contours are at an antenna temperature of $-50 \mathrm{~K} \mathrm{~km} \mathrm{~s}{ }^{-1}$ in $\mathrm{H} \mathrm{I}$ $21 \mathrm{~cm}$ to show the absorption cavities. The large yellow and cyan circles indicate $\eta$ Carinae and the center of the Trumpler 14 cluster. The yellow dots denote $\mathrm{O}-$ and B-type stars (Alexander et al. 2016).

suggests that the dense structures of Tr 14/Carina I are mostly within the -32 to $-5 \mathrm{~km} \mathrm{~s}^{-1}$ velocity range.

\subsection{Channel Maps of [C II] $158 \mu \mathrm{m}$, CO 1-O, H I $21 \mathrm{~cm}$, and $H 92 \mathrm{\alpha}$}

We compare the [C II] $158 \mu \mathrm{m}$ and ${ }^{12} \mathrm{CO} 1-0$ emission to the $\mathrm{H} 92 \alpha$ emission in order to investigate the distribution of the ionized ISM in the $\operatorname{Tr} 14 /$ Carina I region (right column of Figure 3 and channel maps in Appendix B). We find that ionized gas is distributed slightly asymmetrically between $\mathrm{Tr}$ 16 and $\operatorname{Tr} 14$ in velocity space. The H92 $\alpha$ emission near $\operatorname{Tr} 16$ and $\eta$ Carinae spans -60 to $+15 \mathrm{~km} \mathrm{~s}^{-1}$, while the $\mathrm{H} 92 \alpha$ emission near $\operatorname{Tr} 14$ is found from -40 to $+10 \mathrm{~km} \mathrm{~s}^{-1}$. This suggests that the $\mathrm{HII}$ regions near $\operatorname{Tr} 16$ and $\operatorname{Tr} 14$ have different dynamics or spatial distributions. The spatial distribution of the $\mathrm{H} 92 \alpha$ emission has intensity peaks at two different locations: one is the Keyhole Nebula, and the other is Carina I-E, which are both dense clouds near $\eta$ Carinae and $\mathrm{Tr}$ 14. We see the brightest intensities toward the dense clouds rather than the inner $\mathrm{H}$ II region of $\mathrm{Tr} 14$ because the column densities of both the electrons and hydrogen atoms near the dense clouds are higher. The H92 $\alpha$ emission is extended in the $\mathrm{CNC}$, indicating that the ionized gas is widely distributed.

We first compare the H I $21 \mathrm{~cm}$ emission to the $\mathrm{H} 92 \alpha$, [C II] $158 \mu \mathrm{m}$, and ${ }^{12} \mathrm{CO} 1-0$ emission to probe the distribution of the neutral medium to ionized and molecular media. The largescale structure of $\mathrm{HI}$ in the CNC is discussed in Rebolledo et al. (2017), so we focus on the small-scale structures $(<20 \mathrm{pc})$ of the H I emission within the $\operatorname{Tr} 14 /$ Carina I region.

We find that there are cavities in the H I channel maps due to absorption features in the $\mathrm{H}$ I spectra, as shown in Rebolledo et al. (2017). Comparing the cavity to the [C II] and CO emission, we find that the cavities have a strong correlation with $\mathrm{CO}$ and [C II] in PPV space. In position space, we see that the western portion of the cavity coincides with Carina I-E, while the eastern portion of the cavity coincides with the Keyhole Nebula. In velocity space, the H I cavities are at two different velocity ranges: one at -50 to $-20 \mathrm{~km} \mathrm{~s}^{-1}$ and the other at -10 to $0 \mathrm{~km} \mathrm{~s}^{-1}$. The $\mathrm{CO}$ gas 
is at LSR velocities between the two velocity ranges of the H I cavities $\left(-30\right.$ to $\left.-5 \mathrm{~km} \mathrm{~s}^{-1}\right)$. These observations indicate that the neutral atomic medium is spatially associated with the cold molecular clouds but has different kinematics with respect to the dense molecular gas (e.g., cloud dispersal through stripping and photoevaporation).

\subsection{Spectra of CO 1-O, [C II] $158 \mu \mathrm{m}, \mathrm{HI} 21 \mathrm{~cm}, \mathrm{H} 92 \alpha$, and Optical Lines}

To study the ISM structure, we select six positions representative of the $\mathrm{H}$ II region in the $\mathrm{CNC}$, the ionization front of the large CO cloud to the west of $\operatorname{Tr} 14$, and molecular regions (Figure 4, numbers 1-6). We analyze the spectra including ${ }^{13} \mathrm{CO} 1-0,{ }^{12} \mathrm{CO} 1-0$ (Rebolledo et al. 2016), [C II] $158 \mu \mathrm{m}, \mathrm{H}$ I $21 \mathrm{~cm}$ (Rebolledo et al. 2017), H92 $\alpha$, and optical lines of nitrogen and hydrogen (Damiani et al. 2016).

Panels 1, 5, and 6 in Figure 4 present the spectra toward the three positions including the center of $\operatorname{Tr} 14$, the middle position between $\operatorname{Tr} 14$ and $\eta$ Carinae, and $\eta$ Carinae, respectively. We analyze these spectra to trace the kinematics of the $\mathrm{H}$ II region because positions 1 and 6 are the centers of the H II regions formed by $\operatorname{Tr} 14$ and $\operatorname{Tr} 16$, respectively, and position 5 is at the interface of the two $\mathrm{H}$ II regions. We find three common features among the spectra of $\mathrm{H} 92 \alpha, \mathrm{H} \alpha$, and [N II] $6548 \AA$ A: wide velocity ranges compared to $\mathrm{CO}$ and [C II], double intensity peaks, and long tails toward negative velocities. The $\mathrm{H} 92 \alpha, \mathrm{H} \alpha$, and [N II] $6548 \AA$ spectra at all three positions cover velocities from -80 to $+30 \mathrm{~km}^{-1}$. These velocity ranges are at least a factor of 2 larger than those in the other three positions representing PDRs and ionization fronts.

We find double peaks in the $\mathrm{H} 92 \alpha, \mathrm{H} \alpha$, and [N II] $6548 \AA$ profiles at positions 1,5 , and 6 . The blueshifted intensity peaks are at -40 to $-30 \mathrm{~km} \mathrm{~s}^{-1}$, and the redshifted intensity peaks are at -5 to $+5 \mathrm{~km} \mathrm{~s}^{-1}$. As discussed in Damiani et al. (2016), the double intensity peaks likely indicate the red- and blueshifted boundaries/shells of the H II region, since their emission measure is expected to be the highest toward the ionization front due to photoevaporation (e.g., Krumholz et al. 2007). We find that the line widths of the peaks are significantly larger $\left(>30 \mathrm{~km} \mathrm{~s}^{-1}\right.$ for $\mathrm{H} \alpha$ and $>25 \mathrm{~km} \mathrm{~s}^{-1}$ for [N II] $6548 \AA)$ than the thermal broadening $\left(21 \mathrm{~km} \mathrm{~s}^{-1}\right.$ for $\mathrm{H} \alpha$ and $5.7 \mathrm{~km} \mathrm{~s}^{-1}$ for [N II] $6548 \AA$ at at $10,000 \mathrm{~K}$ ), indicating the presence of considerable dynamical motions, such as expansion of the H II regions and turbulence. We also find that the blueshifted portions of the spectra $\left(<-20 \mathrm{~km} \mathrm{~s}^{-1}\right)$ typically have long tails toward negative velocities. For example, in the spectra at position 6 ( $\eta$ Carinae), we see that the blueshifted intensity peak is at $-40 \mathrm{~km} \mathrm{~s}^{-1}$ and the profile extends to $-80 \mathrm{~km} \mathrm{~s}^{-1}$, while the redshifted intensity peak is at $0 \mathrm{~km} \mathrm{~s}^{-1}$ and the profile extends only to $+20 \mathrm{~km} \mathrm{~s}^{-1}$. We see similar profiles toward positions 1 and 5 but with smaller separations between the two intensity peaks and shorter tails compared to the spectra at position 6 . The long tails on the negative velocity side of the spectra indicate that the blueshifted portion of the $\mathrm{H}$ II region is likely larger and expanding faster than the redshifted portion. Considering that there is almost no blueshifted H I $21 \mathrm{~cm}$ emission and that most of the high-mass stars show low extinctions in $\operatorname{Tr} 14$ and $\operatorname{Tr} 16$, it appears that the blueshifted portion of the $\mathrm{H}$ II region has burst through the dense gas and is freely expanding toward us, while the redshifted portion of the $\mathrm{H}$ II region is confined by an $\mathrm{HI}$ cloud, similar to the Champagne model (Tenorio-Tagle 1979).
Comparing the spectra toward positions 1,5 , and 6 , we find that there is $\mathrm{CO}$ emission toward positions 1 ( $\operatorname{Tr} 14)$ and 5 (a middle position between $\operatorname{Tr} 14$ and $\eta$ Carinae), while we do not find any significant $\mathrm{CO}$ emission toward position 6 ( $\eta$ Carinae). This suggests that the $\mathrm{CO}$ clumps in $\operatorname{Tr} 14$ are still confined and interacting with the $\mathrm{H}$ II region, while $\mathrm{Tr} 16$ has mostly cleared out nearby dense structures, except for the $\mathrm{CO}$ cloud east of $\eta$ Carinae, which is consistent with their ages (Walborn 1973; Morrell et al. 1988; Vazquez et al. 1996; Smith \& Brooks 2008; Rochau et al. 2011). We find that the $\mathrm{CO}$ emission at positions 1 and 5 is at a similar LSR velocity of $-18 \mathrm{~km} \mathrm{~s}^{-1}$, while the LSR velocities of the double peaks in $\mathrm{H} 92 \alpha, \mathrm{H} \alpha$, and [N II] $6548 \AA$ are considerably different toward the two positions. The trend is almost the same throughout the entire $\operatorname{Tr} 14$ region. This may indicate that the $\mathrm{CO}$ clouds at $-18 \mathrm{~km} \mathrm{~s}^{-1}$ are beyond the $\mathrm{H}$ II boundary without getting much acceleration by the expanding $\mathrm{H}$ II gas yet.

Position 2 shows the spectra toward a PDR in the north of $\mathrm{Tr}$ 14 (PDR N in Figure 1). This region is considered to be behind $\operatorname{Tr} 14$, since it shows bright emission on its entire surface in $\mathrm{H} \alpha$ and $8 \mu \mathrm{m}$. We find ${ }^{12} \mathrm{CO}$ and [C II] emission around $-20 \mathrm{~km} \mathrm{~s}^{-1}$, indicating that the CO clump has a PDR. We do not find any significant ${ }^{13} \mathrm{CO}$ emission toward this position, indicating that the $\mathrm{CO}$ clump may not have high column density. We also see emission of $\mathrm{H} 92 \alpha, \mathrm{H} \alpha$, and [N II] $6548 \AA$. However, the profiles of those lines show only a single intensity peak with a long tail toward negative velocities. We may not see the double peaks, since this clump is close to the edge of the $\mathrm{H}$ II region and the expanding $\mathrm{H}$ II gas motions are mostly tangential to our LOS. The long tail of the line profile toward to negative velocities may be due to the expansion of the $\mathrm{H}$ II region toward us.

Position 3 is toward an ionization front of the dense $\mathrm{CO}$ wall in the west of $\operatorname{Tr} 14$. We find multiple components of ${ }^{12} \mathrm{CO}$ emission along the LOS and a single component of ${ }^{13} \mathrm{CO}$ emission associated with the strongest ${ }^{12} \mathrm{CO}$ component. We find relatively broad [C II] emission coinciding with the $\mathrm{CO}$ components around $-20 \mathrm{~km} \mathrm{~s}^{-1}$. The $\mathrm{H} 92 \alpha, \mathrm{H} \alpha$, and [N II] $6548 \AA$ lines show different profiles, but the velocities of their intensity peaks are around $-18 \mathrm{~km} \mathrm{~s}^{-1}$, which is near the velocity of the $\mathrm{CO}$ and $[\mathrm{C} \mathrm{II}]$ intensity peaks. This is likely due to the high emission measure of the ionized gas near the PDRs of the CO clumps. We find skewed profiles toward negative velocities as similar as the spectra toward $\operatorname{Tr} 14$, which is likely related to the $\mathrm{H}$ II region expanding toward us.

Position 4 is toward Carina I-E, where we observed the brightest $\mathrm{CO}$ and $[\mathrm{C} \mathrm{II}]$ emission. We find that there are at least two dense CO clumps along the LOS. The CO component at $-23 \mathrm{~km} \mathrm{~s}^{-1}$ is likely in front of $\operatorname{Tr} 14$, since we see it as a dark clump in contrast against a bright $\mathrm{HI}$ background, but it also has bright $[\mathrm{C} \mathrm{II}]$ emission, which indicates that there is a PDR. The entire surface of Carina I-E has significant $\mathrm{H} \alpha$ and $8 \mu \mathrm{m}$ emission, which confirms that we see the PDR of Carina I-E largely face-on but from the back (nonilluminated) side. Another CO component is at $-12 \mathrm{~km} \mathrm{~s}^{-1}$. This component has significantly brighter [C II] emission with respect to the $\mathrm{CO}$ emission. The $\mathrm{H} 92 \alpha$ line includes both of these velocities. The $\mathrm{H} \alpha$ and $[\mathrm{N} \mathrm{II]} 6548 \AA$ lines show single intensity peaks and have their peaks at $-25 \mathrm{~km} \mathrm{~s}^{-1}$, which is different from the double intensity peaks in other positions. This may be mainly due to high optical depth toward dense clumps and obscuring the other intensity peaks. These observations indicate that the component at $-12 \mathrm{~km} \mathrm{~s}^{-1}$ is an $\mathrm{H}$ II region interacting 

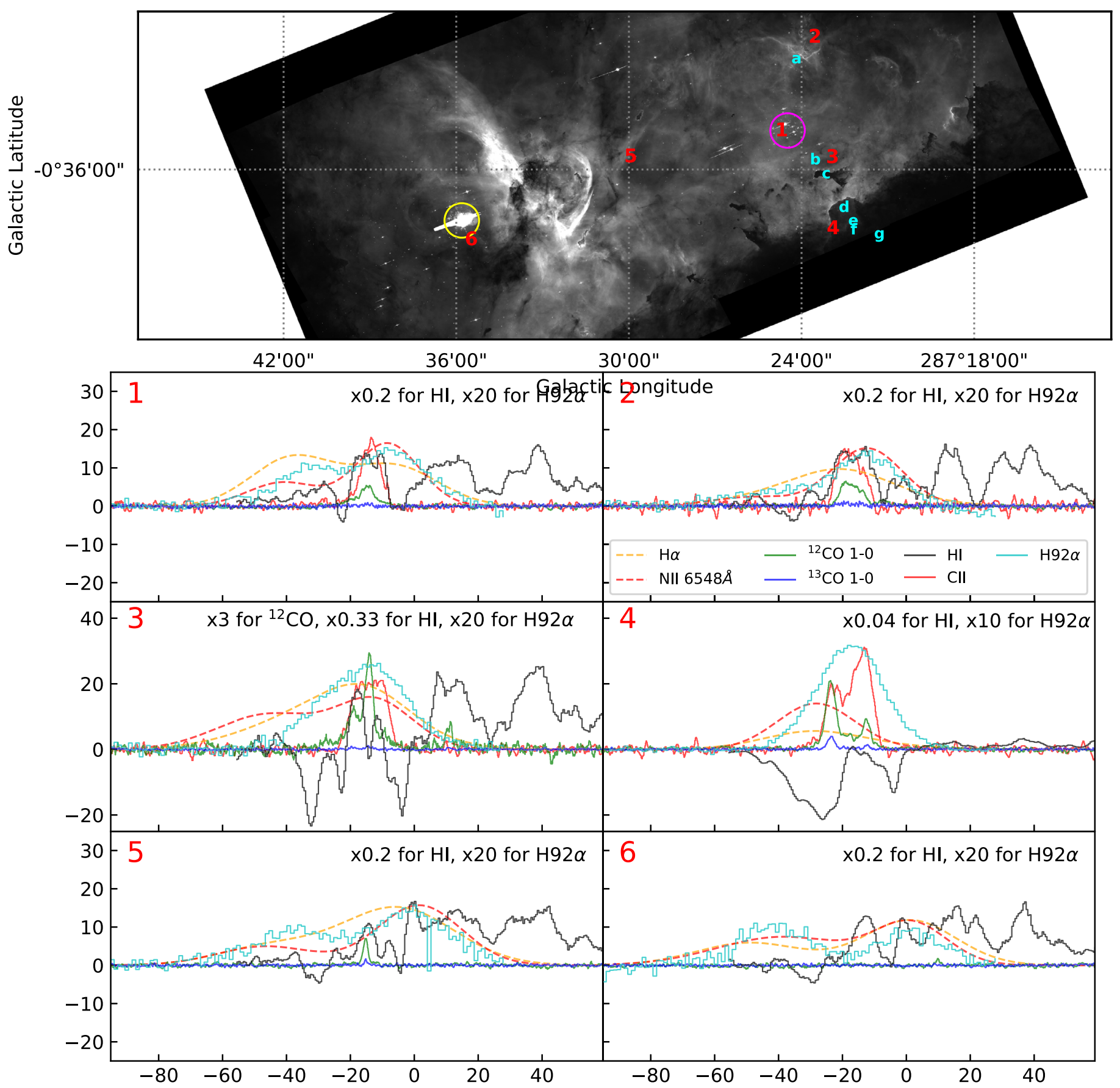

Figure 4. Top panel: six positions employed for comparison of spectra indicated by numbers and seven positions adopted for PDR modeling indicated by letters on the $\mathrm{H} \alpha$ image. The yellow and magenta circles denote $\eta$ Carinae and $\operatorname{Tr} 14$. Bottom panels: spectra of [C II] $158 \mu \mathrm{m},{ }^{12} \mathrm{CO}$ and ${ }^{13} \mathrm{CO} 1-0$ (Rebolledo et al. 2016), $\mathrm{H} \mathrm{I}$ $21 \mathrm{~cm}$ (Rebolledo et al. 2017), H92 $\alpha$, and H $\alpha$ and [N II] $6548 \AA$ (Damiani et al. 2016) toward six selected positions in the Tr 14/Carina I region. The $x$-axis is the LSR velocity in $\mathrm{km} \mathrm{s}^{-1}$. The $y$-axis is the main beam temperature in Kelvin for [C II] $158 \mu \mathrm{m},{ }^{12} \mathrm{CO} 1-0$, and ${ }^{13} \mathrm{CO} 1-0$. For $\mathrm{H} \mathrm{I} 21 \mathrm{~cm}$ and $\mathrm{H} 92 \alpha$, the intensities are in units of Kelvin but with a scaling factor applied to displayed spectra written in each panel. The $y$-axis is the relative intensity for the optical lines. The H $\alpha$ and [N II] $6545 \AA$ are the best-fit models to the Gaia Sky spectra toward the $\mathrm{CNC}$, while the other spectra are the observed spectra.

with a $\mathrm{CO}$ cloud. Comparing to positions 1,5 , and 6 , we see that the $\mathrm{H} 92 \alpha, \mathrm{H} \alpha$, and [N II] $6548 \AA$ lines at position 4 have narrower velocity ranges and single intensity peaks, while the line width $\left(\sim 27 \mathrm{~km} \mathrm{~s}^{-1}\right.$ for $\left.\mathrm{H} 92 \alpha\right)$ is still significantly broader than the thermal line width $\left(21 \mathrm{~km} \mathrm{~s}^{-1}\right.$ at $\left.10,000 \mathrm{~K}\right)$. It is likely that the $\mathrm{H} \mathrm{II}$ region is in between dense clouds (foreground and background), or it may be near the edge of the $\mathrm{H}$ II region, where we would not see the expansion of the H II region along the LOS.
The HI $21 \mathrm{~cm}$ profiles toward positions 3 and 4 have complicated features including both emission and absorption. In the $\mathrm{CNC}$, we find that there are two types of $\mathrm{HI}$ absorption features. One is $\mathrm{HI}$ absorption at the same velocity as ${ }^{13} \mathrm{CO}$ emission. This is likely due to cold $\mathrm{HI}$ gas within the dense $\mathrm{CO}$ clumps, which may still have a relatively high $\mathrm{HI}$ column density due to high total column density in a CO clump combined with a modest fractional abundance of $\mathrm{HI}$ due to a 
relatively young age and incomplete conversion to $\mathrm{H}_{2}$ (Wakelam et al. 2017, and references therein). The absorption feature associated with the ${ }^{13} \mathrm{CO}$ intensity peak toward position 3 suggests that there is cold $\mathrm{HI}$ gas within the molecular clumps. The other absorption features are the ones that are red- or blueshifted relative to the ${ }^{12} \mathrm{CO}$ components. One possible explanation is evaporation or stripping from a CO clump by extreme radiation, since the photoevaporating or radiationstripped H I gas from the CO clumps can appear as absorption against the free-free emission background produced by the $\mathrm{H}$ II region. We find that the velocity differences between the absorption features and the CO components in the Tr 14/Carina I region are typically $\leqslant 10 \mathrm{~km} \mathrm{~s}^{-1}$. These velocity differences are similar to the photoevaporation or radiation-stripping velocity from the $\mathrm{CO}$ clouds predicted by numerical simulations (e.g., Bertoldi 1989; Bertoldi \& McKee 1990; Lefloch \& Lazareff 1994; Mellema et al. 1998; McLeod et al. 2016). In addition, the spatial distribution of the absorption features coincides with the dense clouds (e.g., Carina I-E/Carina I-W and Keyhole Nebula) in the CNC. It is thus likely that the absorption features are due to the dynamics related to the cloud dispersal by photoevaporation and radiation stripping.

One common feature in $\mathrm{H} \mathrm{I} 21 \mathrm{~cm}$ lines at all positions is the asymmetric distribution of $\mathrm{H}$ I gas in velocity space seen in the channel maps. Looking at details of $\mathrm{H}$ I distribution in velocity, we find abundant neutral hydrogen at LSR velocities larger than $-20 \mathrm{~km} \mathrm{~s}^{-1}$ at all six positions, while we do not see significant $\mathrm{HI}$ emission at the LSR velocity smaller than $-20 \mathrm{~km} \mathrm{~s}^{-1}$. We find this behavior at all positions around the CNC. Considering the wide distribution of the H I emission at the same LSR velocity, we think that the $\mathrm{HI}$ cloud at $>-20 \mathrm{~km} \mathrm{~s}^{-1}$ confines the redshifted portion of the $\mathrm{H}$ II region. This agrees with the consistent LSR velocity of the CO components at $-18 \mathrm{~km} \mathrm{~s}^{-1}$ across the Tr 14/Carina I region.

\section{Modeling PDRs and H II Gas in the Tr 14/Carina I Region}

A number of authors have previously applied PDR models to IR data of the Tr 14/Carina I region (Brooks et al. 2003; Mizutani et al. 2004; Oberst et al. 2011; Okada et al. 2013; Wu et al. 2018). Typically, the observations and models included several of the following: [C II] $158 \mu \mathrm{m}$; [O I] 63, $145 \mu \mathrm{m}$; [C I] 369, $609 \mu \mathrm{m}$; ${ }^{12} \mathrm{CO}$ low-to-mid-J transitions; and IR continuum. Several authors pointed out that the [O I] $63 \mu \mathrm{m}$ (and even possibly [C II] $158 \mu \mathrm{m}$; see Mizutani et al. 2004 and Wu et al. 2018) could suffer self-absorption and therefore was not used in the comparisons of observations to their PDR models. Within a $10^{\prime}$ or roughly $7 \mathrm{pc}$ projected distance from $\operatorname{Tr} 14$, Brooks et al. (2003) and Oberst et al. (2011) found rough matches with constant density PDR models that had hydrogen nucleus densities of $\sim 300-3 \times 10^{4} \mathrm{~cm}^{-3}$ and FUV fields $G_{0} \sim 600-10^{4}$. Kramer et al. (2008), using the clumpy KOSMA- $\tau$ model, found $G_{0} \sim 500-5 \times 10^{3}$ and somewhat higher ensemble average densities of $2 \times 10^{5} \mathrm{~cm}^{-3}$. Given that the FUV luminosity of $\operatorname{Tr} 14$ is roughly $2 \times 10^{6} L_{\odot}$, this range of $G_{0}$ corresponds to distances of 2.1-8.5 pc if there is insignificant extinction of FUV inside the $\mathrm{H}$ II region. The molecular ridge to the southwest of $\mathrm{Tr}$ 14 is about $2.3 \mathrm{pc}$ in projected distance, so the derived $G_{0}$ values are in rough agreement with the likely geometry of the neutral gas around the $\operatorname{Tr} 14 \mathrm{H}$ II region.

Wu et al. (2018) applied constant thermal pressure Meudon PDR models (Le Petit et al. 2006) and found a range of
$P_{\text {th }} \sim 3 \times 10^{7}-3 \times 10^{8} \mathrm{~K} \mathrm{~cm}^{-3}$ and $G_{0} \sim 3 \times 10^{3}-5 \times 10^{4}$, somewhat higher values than previous authors. We discuss these high values below. Our main thrust in this section is to understand and discuss the interesting relation between the thermal pressure in the PDR and the incident FUV flux found by Wu et al. (2018). Wu et al. (2018) used PACS observations of $\mathrm{CO}$ (up to $J=13-12$ ) and both CI fine-structure transitions to find best-fit PDR models for each pixel in a large map of Car I-E, Car I-S, and Car I/II. The main free parameters in the models are $P_{\text {th }}$ and $G_{0}$ (and, to a lesser extent, the beam filling factor and total column density through the PDR layer), and they used the best fit to each pixel to generate a large number of $P_{\text {th }}$ and $G_{0}$ pairs. ${ }^{20}$ From the observations and the modeling of each pixel, the empirical relation is

$$
P_{\text {th }}=2.7 \times 10^{4} G_{0}^{0.9} \mathrm{~K} \mathrm{~cm}^{-3} \text {. }
$$

$\mathrm{Wu}$ et al. (2018) did not quote errors in this fit, but their Figure 13 suggests that the errors could be significant.

In this section, we first analytically derive the expected relation of the applied pressure to the PDR, $P_{\mathrm{PDR}}$, to $G_{0}$ using the Strömgren relations for $\mathrm{HII}$ regions and the relative strengths of the EUV luminosity $\Phi_{\mathrm{EUV}}$ and the FUV luminosity $\Phi_{\mathrm{FUV}}$ from the $\mathrm{OB}$ association. We note that, even in steady state, the applied pressure, $P_{\mathrm{PDR}}$, may differ from the thermal PDR pressure, $P_{\mathrm{th}}$, because of other sources of PDR pressure support (see below). We compare the relation $P_{\mathrm{PDR}}$ to $G_{0}$ with the Wu et al. (2018) semi-empirical relation of $P_{\text {th }}$ to $G_{0}$ (Equation (1)). We apply our PDR models to the integrated intensities from STO2 [C II] observations, as well as the literature values of $\mathrm{CO}(J=1-0)$ (Rebolledo et al. 2016), CO ( $J=4-3$ and 7-6) (Kramer et al. 2008), the CI fine-structure lines (Kramer et al. 2008), [O I] 63 and $145 \mu \mathrm{m}$ (Mizutani et al. 2004; Oberst et al. 2011; Wu et al. 2018), and the IR continuum (Preibisch et al. 2012) and find $P_{\text {th }}$, $G_{0}$ pairs consistent with observations in a manner similar to $\mathrm{Wu}$ et al. (2018). We use [N II] observations (Oberst et al. 2011) when available to estimate the [C II] emission from the ionized $\mathrm{H}$ II gas. We find the $[\mathrm{O} \mathrm{I}]$ and $[\mathrm{C} \mathrm{II}]$ lines helpful in constraining our fits, although we place less weight on the [O I] $63 \mu \mathrm{m}$ integrated intensity fitting ([O I] $63 \mu \mathrm{m}$ can suffer significant self-absorption) except to ensure that the PDR model intensity is at least as bright as observed. We then also compare our PDR model results with our derived analytical relation.

\subsection{Analytic Derivation of Dependence of $\mathrm{P}_{P D R}$ on $\mathrm{G}_{O}$}

The basic Strömgren relation for an H II region with no wind cavity and assuming constant electron density $n_{e}$ inside the $\mathrm{H} \mathrm{II}$ region is

$$
\Phi_{\mathrm{EUV}} f_{\mathrm{gas}}=\frac{4}{3} \pi \alpha_{r} n_{e}^{2} d^{3},
$$

where $\Phi_{\mathrm{EUV}}$ is the EUV photon luminosity of the source, $f_{\text {gas }}$ is the fraction of the EUV absorbed by recombinations in the $\mathrm{H} \mathrm{II}$ gas (and not the dust in the H II region), $d$ is the Strömgren radius of the $\mathrm{H}$ II region (and also the distance from the UV source to a PDR lying just outside the ionization front), and $\alpha_{r}$ is the recombination coefficient of electrons with protons in the $\mathrm{H}$ II region. Using the on-the-spot assumption, so that only

\footnotetext{
${ }^{20}$ In fact, Wu et al. (2018) used Mathis units and gave their FUV fit in these units, $G_{\mathrm{UV}}$. In the Habing units that we use, the relation between the two is $G_{0}=1.3 G_{\mathrm{Uv}}$. In this section, we convert the $\mathrm{Wu}$ et al. (2018) results to $G_{0}$ units.
} 
recombinations to the excited levels are counted, we take $\alpha_{r}=2.6 \times 10^{-13} \mathrm{~cm}^{3} \mathrm{~s}^{-1}$, assuming the $\mathrm{H}$ II region temperature is $T=10^{4} \mathrm{~K}$. We define a proportional number $f$ such that

$$
\Phi_{\mathrm{FUV}}=f \Phi_{\mathrm{EUV}},
$$

where $\Phi_{\mathrm{FUV}}$ is the FUV photon luminosity in the wavelength range 912-2000 ̊. Typically, for large and young OB associations like $\operatorname{Tr} 14$, with a number of very hot and earlytype stars, $f \sim 1$. For $\operatorname{Tr} 14$, Smith (2006) found $\Phi_{\mathrm{EUV}}=2.2 \times$ $10^{50}$ EUV photons $\mathrm{s}^{-1}$ and $L_{\mathrm{FUV}}=2.0 \times 10^{6} L_{\odot}$. The latter luminosity can be approximately converted to a photon luminosity by assuming the average energy of an FUV photon is $10 \mathrm{eV} ; \Phi_{\mathrm{FUV}}=4.8 \times 10^{50}$ FUV photons $\mathrm{s}^{-1}$. This makes $f=\Phi_{\mathrm{FUV}} / \Phi_{\mathrm{EUV}}=2.18$.

The incident FUV flux on the PDR just outside the H II region is then written as

$$
G_{0}=\frac{\Phi_{\mathrm{FUV}} f_{\mathrm{PDR}}}{4 \pi d^{2} F_{0}}
$$

where $F_{0} \simeq 10^{8}$ photons $\mathrm{cm}^{-2} \mathrm{~s}^{-1}$ is the FUV flux appropriate for $G_{0}=1$, and $f_{\mathrm{PDR}}$ is the fraction of FUV photons that escape dust absorption in the $\mathrm{H}$ II region: $f_{\mathrm{PDR}} \simeq f_{\text {gas }}$. Here we assume that the PDR is a spherical shell that surrounds the H II region or a cloud surface with a size greater than the distance $d$ to the UV source. Once we find $G_{0}$ and $f_{\mathrm{PDR}}$ by comparing PDR models with observations, this equation can be used to determine $d$, the distance of the PDR from $\operatorname{Tr} 14$. The thermal pressure in the $\mathrm{H}$ II region is given by

$$
P_{\mathrm{H} \mathrm{II}}=2 n_{e} T \text {, }
$$

where we have assumed that for each electron, there is one positive charge carrier, either $\mathrm{H}^{+}$or $\mathrm{He}^{+}{ }^{21}$ Assuming that $T=10^{4} \mathrm{~K}$ in the H II region, we use Equations (2)-(5) to obtain

$$
P_{\mathrm{H} \mathrm{II}}=2.3 \times 10^{4} f^{-3 / 4} \Phi_{51}^{-1 / 4} G_{0}^{3 / 4} \mathrm{~K} \mathrm{~cm}^{-3},
$$

where $\Phi_{51}=\Phi_{\mathrm{EUV}} / 10^{51}$ photons s$^{-1}$. Note that the dependence of $P_{\mathrm{H} \mathrm{II}}$ on $G_{0}\left(P_{\mathrm{H} \mathrm{II}} \propto G_{0}^{0.75}\right)$ is close to but not quite the same as the empirical relation $\left(P_{\text {th }} \propto G_{0}^{0.9}\right)$ given by Wu et al. (2018). We also note that the simplest assumption for a confined H II region with a PDR that surrounds it is that $P_{\mathrm{H} \mathrm{II}}=P_{\mathrm{PDR}}$. However, the $\mathrm{H}$ II region around $\mathrm{Tr} 14$ does not appear confined but rather is a blister $\mathrm{H}$ II region that is expanding away from the GMC. In this case, there is additional pressure on the PDR caused by the ram pressure of the photoevaporating $\mathrm{H}$ II gas off the PDR surface. This additional pressure is of the order of the thermal pressure (Equation (A4) in Gorti \& Hollenbach 2002), so that the applied pressure on the PDR $P_{\mathrm{PDR}} \simeq 2 P_{\mathrm{H} \mathrm{II}}$, where $P_{\mathrm{H} \text { II }}$ is the thermal pressure in the outflowing ionized gas from the PDR surface.

The normalization constant in the $P_{\mathrm{PDR}}$ versus $G_{0}$ relation can be compared to the normalization constant in Wu et al. if we specify the Smith (2006) values of $f$ and $\Phi_{\mathrm{EUV}}$ in the $\operatorname{Tr} 14$ cluster of $\mathrm{OB}$ stars and assume $P_{\mathrm{PDR}}=2 P_{\mathrm{H} \mathrm{II}}$,

$$
P_{\mathrm{PDR}}=3.7 \times 10^{4} G_{0}^{0.75} \mathrm{~K} \mathrm{~cm}^{-3} .
$$

\footnotetext{
${ }^{21}$ Note that if $\mathrm{He}$ is doubly ionized, the factor 2 is slightly smaller, but we shall ignore that small correction.
}

This equation appears very similar to the empirical relation $P_{\text {th }}$ versus $G_{0}$ found by Wu et al. (2018). We need, however, to test it in the region of applicability of the $\mathrm{Wu}$ et al. relation. The relation found by $\mathrm{Wu}$ et al. (2018) was for PDRs where $G_{0} \sim 3 \times 10^{3}-5 \times 10^{4}$. The Wu et al. relation (Equation (1)) gives $P_{\mathrm{th}}=3.6 \times 10^{7}$ and $4.6 \times 10^{8} \mathrm{~K} \mathrm{~cm}^{-3}$ for $G_{0}=3 \times$ $10^{3}$ and $5 \times 10^{4}$, respectively, while our analytic relation (Equation (7)) gives $P_{\mathrm{PDR}}=1.5 \times 10^{7}$ and $1.2 \times 10^{8} \mathrm{~K} \mathrm{~cm}^{-3}$, respectively. Thus, the analytic solution is indeed quite close, perhaps a factor of 3-4 lower than the $P_{\text {th }}$ found by the best-fit PDR models in $\mathrm{Wu}$ et al. (2018). ${ }^{22}$

We again stress here that $P_{\mathrm{th}}$ is the thermal pressure in the PDR gas, since PDR models often hold the thermal pressure constant, and it is this pressure that PDR modelers, including this paper, plot in their figures. With the exception of the applied pressure $P_{\mathrm{PDR}}$ in clumps discussed below, Equation (7) provides an upper limit to $P_{\text {th }}$ because it assumes that the applied pressure to the neutral region is balanced by the thermal pressure of the PDR gas. If magnetic pressure supports the PDR gas, then, for a given $G_{0}, P_{\mathrm{th}}<P_{\mathrm{PDR}}$, since the sum of thermal pressure and magnetic pressure should equal the applied pressure in steady state. Since the neutral gas around $\mathrm{H}$ II regions and in clumps inside $\mathrm{HII}$ regions has been pressurized and compressed by the expanding, high-temperature $\mathrm{H}$ II gas, one might expect larger ratios of magnetic pressure to thermal pressure in the compressed gas than in ambient gas because magnetic pressure generally increases more rapidly with compression than thermal pressure.

We show below that the PDR code provides 10 fits that have filling factors of essentially unity, suggesting a shell or partial shell just outside the H II region. However, one fit requires a beam filling factor significantly smaller than unity. Such a small beam filling factor suggests neutral clumps inside the extended H II region. Opaque clumps are certainly seen in the optical images, and clumps appear in the maps of $\mathrm{CO}$ we discussed above and are inferred in the Wu et al. (2018) study. The EUV evaporating clumps inside an $\mathrm{H}$ II region have a different relation of $P_{\mathrm{PDR}}$ to $G_{0}$. In fact, another variable is introduced: the radius $R$ of the clump. If $R>d$, where $d$ is the distance of the clump from the EUV source $\operatorname{Tr} 14$, then the solution given in Equation (7) still applies. However, if $R \ll d$, then we have a small EUV evaporating clump inside the extended H II region. A very similar computation to that described above applies, except that the incident EUV flux is absorbed by $\mathrm{H}$ atoms that have recombined in the evaporating flow off of the clump (see Bertoldi \& McKee 1990, for a detailed analysis). Assuming that the flow is ejected at $\sim 10 \mathrm{~km} \mathrm{~s}^{-1}$, the thermal speed of the $10^{4} \mathrm{~K}$ ionized gas at the surface of the clump, one finds that, analogous to the steps above for standard H II regions, the applied pressure to the clump is

$$
P_{\mathrm{PDR}} \simeq 4 T_{\mathrm{H} \mathrm{II}}\left(\frac{3 F_{0} G_{0}}{\alpha_{r} R f}\right)^{1 / 2},
$$

where $T_{\mathrm{H} \text { II }} \sim 10^{4} \mathrm{~K}$ is the temperature of the ionized gas streaming off the clump, cgs units are used, and the pressure is in units of $\mathrm{K} \mathrm{cm}^{-3}$. Note that for a fixed $G_{0}$, the pressure now

\footnotetext{
$\overline{22}$ We have checked to see if stellar winds could apply sufficient additional pressure to explain the discrepancy but found, using stellar wind parameters from Smith (2006), that the winds are too weak to explain the factor of $\sim 3-4$.
} 
depends on the clump radius $R$. For $R \ll d$, the derived pressure is higher than the $P_{\mathrm{PDR}}$ given in Equation (7). Small clumps require higher densities at the ionization front in order to absorb the incident EUV, since the characteristic distance $R$ for the EUV to be absorbed is smaller. The higher densities produce higher applied pressures to the PDR. It is possible that some of the higher pressures found by Wu et al. (2018) are caused by clumps along their LOSs. Alternatively, the area mapped by Wu et al. (2018) may have localized sources of UV that can lead to a higher $P_{\mathrm{PDR}}$ for a given $G_{0}$ (see Equation (6)). Another possibility is differences in the chemistry and heating processes in the two PDR codes.

\subsection{PDR Modeling and STO2 [C II] Results}

The analysis of the observations is carried out using a PDR model based on that of Wolfire et al. (2010) and Hollenbach et al. (2012), with additional updates noted in Neufeld \& Wolfire (2016). The models calculate the steady-state chemical abundances and thermal balance gas temperature of a layer of gas of constant thermal pressure, $P_{\text {th }}$, exposed to an FUV radiation field, $G_{0}$, and cosmic-ray ionization rate $\zeta_{\mathrm{CR}}$. The radiation field is measured in units of the interstellar field of Habing integrated between 6 and $13.6 \mathrm{eV}\left(=1.6 \times 10^{-3} \mathrm{erg} \mathrm{cm}^{-2} \mathrm{~s}^{-1}\right)$. To aid in the analysis, we have carried out a grid of models with $G_{0}$ varying in $\log$ steps of 0.25 in the range $-0.5 \leqslant \log G_{0} \leqslant 6.5$ and thermal pressure varying in log steps of 0.25 in the range $2 \leqslant \log P_{\text {th }} /\left(\mathrm{K} \mathrm{cm}^{-3}\right) \leqslant 8$.

The cosmic-ray ionization rate and gas-phase abundances are held fixed as given in Table 2. The model output consists of integrated emission line intensities as functions of $G_{0}$ and $P_{\text {th }}$ for lines that arise in the atomic and molecular gas in the PDR, including [C II] $158 \mu \mathrm{m}, \mathrm{CO} 1-0, \mathrm{CO} 4-3, \mathrm{CO} 7-6$, [C I] $610 \mu \mathrm{m}$, [C I] $370 \mu \mathrm{m}$, [O I] $63 \mu \mathrm{m}$, and [O I] $145 \mu \mathrm{m}$. The PDR models do include self-absorption inside the PDR region, as the lines emerge from the illuminated face. However, they neglect self-absorption by cool foreground clouds or for LOSs that approach the PDR from the nonilluminated (back) side. The [O I] $63 \mu \mathrm{m}$ line has the highest optical depth and is the most prone to this effect. Therefore, in our best fits, we allow the PDR model to somewhat overpredict the integrated [O I] $63 \mu \mathrm{m}$ line. However, in the models presented below, it is never more than a factor of $\sim 2-3$. We emphasize that we do not allow our best-fit models to underpredict the [O I] lines by more than a factor of 2 . We find that when there is overlap, the PACS observational [O I] line intensities (Wu et al. 2018), even when matched to the ISO beam sizes, give higher intensities than those quoted in Oberst et al. (2011). Our models suggest the higher values, and we therefore match our models to the integrated and convolved PACS data when they are available.

We use the Mopra ${ }^{13} \mathrm{CO} J=1-0$ observations to estimate the total column of the PDR slab for each of our models. We take the PDR model temperature at $A_{V}=5$ into the slab to estimate the average temperature of the ${ }^{13} \mathrm{CO}$ in order to translate the integrated optically thin line emission into a column or a $\Delta A_{V}(\mathrm{CO})$ of gas that is both molecular $\mathrm{H}_{2}$ and $\mathrm{CO}$. We assume that the isotopic ${ }^{12} \mathrm{C} /{ }^{13} \mathrm{C}$ ratio is 60 (Szúcs et al. 2014). The PDR model allows us to compute the $\Delta A_{V}$ (atomic), which we define as the $\Delta A_{V}$ from the PDR surface to where the gas is half molecular $\mathrm{H}_{2}$ and half atomic. Similarly, we obtain $\Delta A_{V}$ (dark), which we define as the $\Delta A_{V}$ from where the gas is half molecular to where the optical depth in the ${ }^{12} \mathrm{CO}$ line is unity. The sum of these $\Delta A_{V}$ is the total $A_{V}$ of the PDR slab.

An additional model parameter is the source area filling factor in the telescope beam, $f_{\mathrm{B}}$. In practice, we compute $f_{\mathrm{B}}$ by comparing our model $\mathrm{CO} 1-0$ intensity to the observed intensity and then fit all other lines by matching the ratio of the line to $\mathrm{CO} 1-0$.

Another important model constraint comes from the integrated IR continuum observations. We use the Herschel PACS (70 and $160 \mu \mathrm{m})$ and SPIRE $(250,350$, and $500 \mu \mathrm{m})$ observations (Preibisch et al. 2012) convolved to the STO2 beam size. We fit each SED with a dust optical depth $\tau_{\mathrm{D}}$, dust temperature $T_{\mathrm{D}}$, and emissivity index $\beta$ and integrate under the resultant fit to find the integrated continuum intensity $I_{\mathrm{IR}}$. We assume that this IR continuum arises from the sum of contributions from the PDR and the H II region: $I_{\mathrm{IR}}=I_{\mathrm{PDR}}+I_{\mathrm{H} \mathrm{II}}$. Using theoretical spectra from O stars (Parravano et al. 2003; Malkov 2007), we find that approximately half the dust heating in the PDR is from the FUV photons, and the rest is from photons outside this band. Therefore, $I_{\mathrm{PDR}}=2 \times 1.6 \times 10^{-3} \mathrm{f}_{\mathrm{B}} G_{0} /(4 \pi) \mathrm{erg} \mathrm{cm}^{-2} \mathrm{~s}^{-1} \mathrm{sr}^{-1}$. Our best-fit models provide $G_{0}$ and $f_{\mathrm{B}}$, and the observations provide $I_{\mathrm{IR}}$. We can then estimate $I_{\mathrm{H} \text { II }}=I_{\mathrm{IR}}-I_{\mathrm{PDR}}$.

In the model fits, we vary only $P_{\text {th }}, G_{0}$, and $f_{\mathrm{B}}$, although additional factors such as geometry, abundance variations, and depth of the PDR layer may affect the emitted line intensities. In light of these considerations and possible observational errors, we assume that fits to the observed line and continuum intensities to within factors of 2 are considered to be good fits, although we generally find agreement to better than a factor of 2. Below, we discuss each fit and the maximum difference between the good fit model and the observations.

To estimate the $[\mathrm{C} \mathrm{II}]$ line emission from ionized gas, we rely on the observed [N II] line intensities. The [C II] $158 \mu \mathrm{m} /[\mathrm{N}$ II] $205 \mu \mathrm{m}$ ratio is weakly dependent on electron density varying between 5.1 at the low-density limit and 6.0 at the high-density limit, with a minimum of 3.7 at $n_{e} \approx 50 \mathrm{~cm}^{-3}$. The [C II] $158 \mu \mathrm{m} /[\mathrm{N}$ II $] 122 \mu \mathrm{m}$ ratio has a stronger dependence on $n_{e}$ varying monotonically between 0.62 (at the high-density limit, roughly $n_{e}>1000 \mathrm{~cm}^{-3}$ ) and 9.7 (at the low-density limit, roughly $n_{e}<1 \mathrm{~cm}^{-3}$ ). These ratios are calculated assuming the gas-phase abundances of $\mathrm{C}^{+}$and $\mathrm{N}^{+}$to be $1.6 \times 10^{-4}$ (Sofia et al. 2004) and $7.5 \times 10^{-5}$ (Meyer et al. 1997), respectively. The electron collision strengths are from Tayal (2011) for [N II] and Tayal (2008) for [C II] for $T=8000 \mathrm{~K}$ gas. We can estimate the electron density from the [N II] $122 \mu \mathrm{m} /[\mathrm{N}$ II] $205 \mu \mathrm{m}$ ratio and then obtain the $[\mathrm{C} \mathrm{II}]$ line intensity from either of the $[\mathrm{N}$ II] lines. This ratio varies from 0.53 in the low-density limit $\left(n_{e} \lesssim 1 \mathrm{~cm}^{-3}\right)$ to 9.7 in the high-density limit $\left(n_{e} \gtrsim 1000 \mathrm{~cm}^{-3}\right)$. If either [N II] line is not observed, an alternative approach to obtain the electron density is to use the best-fit thermal pressure in the PDR. From Equation (5), we find the electron density from

$$
n_{e}=\frac{P_{\mathrm{H} \mathrm{II}}}{\left(\mathrm{K} \mathrm{cm}^{-3}\right)} \frac{1}{2 T}=\frac{P_{\mathrm{PDR}}}{(2 \times 2 \times 10,000)},
$$

where we assume a gas temperature in the ionized gas of $10,000 \mathrm{~K}$ and $P_{\mathrm{PDR}}=2 P_{\mathrm{H} \mathrm{II}}$.

We have selected 10 spectral features from seven positions to analyze in detail (see Figures 4 and 5). These positions are labeled by the letters $\mathrm{a}-\mathrm{g}$ in Figure 4 . The points generally lie along a line from north of the Tr 14 cluster to southwest into the molecular ridge. For each spectrum, we integrate the line profiles over one or two likely velocity components, and we 
associate the (spectrally unresolved) [N II] line intensity (i.e., the ionized gas) with the brighter [C II] component. Often, the observed [C II] line intensity in the bright component is brighter than what can be produced by the neutral PDR emission alone, and the expected contribution from the ionized gas is required to match the observations. When available, we use the theoretically expected ratio of $[\mathrm{N}$ II] to $[\mathrm{C} \mathrm{II}]$ to estimate the [C II] flux from the $\mathrm{H}$ II region. We do not apply a filling factor correction to the ionized gas emission; i.e., we assume the ionized gas fills the beam. For observations with higher angular resolution than the $[\mathrm{C} \mathrm{II}]$ resolution, we convolve the observations to provide the integrated intensity in the [C II] beam. If two velocity components are present along the LOS, then the observed continuum emission arises from the sum of both components. Using Equation (4), we also estimate a crude distance of the PDR layer, $d$, away from Tr 14 using the model $G_{0}$, the FUV luminosity estimate of Smith (2006), and the IR continuum intensities obtained by model and observation. Here we assume that $f_{\mathrm{PDR}} \simeq I_{\mathrm{PDR}} / I_{\mathrm{IR}}$. The following gives a summary of our fits for each point. We follow the summary of each position with a short discussion of these results.

(a) PDR North of $\operatorname{Tr} 14(l, b)=287.403,-0.537$. The spectra indicate two basic velocity features, one from -11 to $-4 \mathrm{~km} \mathrm{~s}^{-1}$ and one from -25 to $-11 \mathrm{~km} \mathrm{~s}^{-1}$. However, the CO 4-3 and 7-6 and the [C I] observations suffer baseline problems and are not reliable, except possibly the $\mathrm{CO}$ 4-3 transition in the first velocity feature, which is fairly strong. We model this first feature because we have reliable CO 1-0, [C II], [O I], and IR continuum observations. We find an excellent fit (the model fits the observation to within a factor of 1.2 for each line and the continuum) to all of these observations with a PDR model with $\log P_{\text {th }}=7.25, \log G_{0}=3.75$, and $f_{\mathrm{B}}=0.1$. The $\triangle A_{V}$ values for the atomic, dark, and molecular CO gas are 1.1, 2.3 , and 4.4, respectively. The beam filling factor suggests a clump or clumps in the beam. If a single clump dominates, then its diameter is about 0.3 of the beam diameter, or radius $R=0.1 \mathrm{pc}$. On the other hand, we may be observing just a portion of a larger cloud. The incident $G_{0}$ suggests a distance from $\operatorname{Tr} 14$ of about $2.5 \mathrm{pc}$. This redshifted component compared to the H II gas (whose emission centers at approximately $-17 \mathrm{~km} \mathrm{~s}^{-1}$ ) may be on the far side of the $\mathrm{H} \mathrm{II}$ region, traveling away from us. As we shall demonstrate below, out of the 10 features modeled, this is the only spectral feature that suggests a small clump in the beam. As seen in the $\mathrm{H} \alpha$ map, this position lies in a direction of unobscured optical emission and may be primarily ionized gas. This is borne out by the strong [C II] emission from the second velocity feature, which likely arises in the ionized gas.

(b) The middle point between Tr 14 and the dust lane (l, b) $=287.392,-0.596$. The spectra suggest a single velocity component: -24 to $-7 \mathrm{~km} \mathrm{~s}^{-1}$. The [C I] observations suffered baseline problems and were not used in this fit, which matched the CO 1-0, 4-3, and 7-6 and the two [O I] lines. The PDR model fit is $\log P_{\text {th }}=5.5, \log G_{0}=2.25$, and $f_{\mathrm{B}} \simeq 0.5$. The $\triangle A_{V}$ values for the atomic, dark, and molecular CO gas are 0.6, 2.2 , and 1.7 , respectively. The ratio of the PDR model flux to the observed flux (henceforth " $\mathrm{m} / \mathrm{o}$ ") ranges from 0.5 for [O I] $63 \mu \mathrm{m}$ to 1.9 for CO 7-6. The observed [N II] scales to predict a [C II] integrated intensity from the $\mathrm{H}$ II region that is a factor of 0.54 of the observed; our PDR model fit only adds about $10 \%$ to the $\mathrm{H}$ II contribution, so the $\mathrm{H}$ II region dominates the [C II] production in this case. The same is true of the IR continuum emission. We find that only $10 \%$ comes from the PDR and the rest from the $\mathrm{H}$ II region. In general, we find that when the $\mathrm{H}$ II region dominates the [C II], it also dominates the IR, suggesting significant extinction of the UV as it traverses the $\mathrm{H}$ II region to the PDR. Taking into account this extinction, we find $d_{\mathrm{PDR}}=4.9 \mathrm{pc}$.

(c) CG South of $\operatorname{Tr} 14(l, b)=287.386,-0.604$. We fit a single velocity component extending from -24 to $-7 \mathrm{~km} \mathrm{~s}^{-1}$. The PDR model fit is $\log P_{\text {th }}=6.0, \log G_{0}=2.5$, and $f_{\mathrm{B}} \simeq 0.5$. The $\Delta A_{V}$ values for the atomic, dark, and molecular $\mathrm{CO}$ gas are $0.5,2.2$, and 2.4, respectively. The PDR fit is based on the $\mathrm{CO},[\mathrm{C}$ I], and [O I] fluxes. For [O I] 63 and $145 \mu \mathrm{m}$, the $\mathrm{m} / \mathrm{o}=1.1$ and 1.3 , respectively. The worst $\mathrm{m} / \mathrm{o}=1.8$ is for CO 7-6. Our model fits the [C II] if we scale the observed [N II] $205 \mu \mathrm{m}$ to estimate [C II] from the $\mathrm{H}$ II region. We find that about $90 \%$ of the $[\mathrm{C} \mathrm{II}]$ arises from the $\mathrm{H}$ II region and $10 \%$ from the PDR. We note here that we have found another test besides [N II] to determine if the [C II] arises mostly from the ionized gas. If the flux ratio $[\mathrm{C} \mathrm{II}] / \mathrm{CO}(1-0) \gtrsim 10^{4}$, then the $\mathrm{H}$ II region dominates. The PDR dominates typically when the ratio is of order 1000-4000 (see also Wolfire et al. 1989). Again, just like the [C II], we find that $15 \%$ of the IR comes from the PDR and the rest from the H II region. Using the implied extinction in the $\mathrm{H}$ II region, we find $d_{\mathrm{PDR}}=4.5 \mathrm{pc}$.

(d) North surface of Carina I-E $(l, b)=287.376,-0.622$. Here the spectra suggest two velocity components. The first component extends from -20 to $-8 \mathrm{~km} \mathrm{~s}^{-1}$. The PDR model fit is $\log P_{\text {th }}=6.0, \log G_{0}=2.0$, and $f_{\mathrm{B}} \simeq 0.6$. The $\Delta A_{V}$ values for the atomic, dark, and molecular $\mathrm{CO}$ gas are $0.08,1.9$, and 1.8, respectively. The PDR model fit is based on the $\mathrm{CO}$ and $[\mathrm{CI}]$ observations. The worst $\mathrm{m} / \mathrm{o}=2.0$ is for $[\mathrm{CI}] 2-1$. The ionized gas is associated with this component, and, scaling from the [N II], we find a match to the [C II] from this component. Only about $8 \%$ comes from this PDR component. Similarly, this component only contributes about $3 \%$ of the IR continuum. As noted below, the IR comes from the other PDR velocity component, with a $10 \%$ contribution from the $\mathrm{H}$ II region. Ignoring extinction in the $\mathrm{HII}$ region in this case, we find $d_{\mathrm{PDR}}=20 \mathrm{pc}$. This gas is somewhat redshifted relative to the ionized gas and may lie behind the $\mathrm{HII}$ region, which apparently extends quite far back in this direction.

The second velocity component extends from -35 to $-20 \mathrm{~km} \mathrm{~s}^{-1}$. The PDR model fit is $\log P_{\mathrm{th}}=7.25, \log G_{0}=$ 3.5 , and $f_{\mathrm{B}}=0.6$, and $d_{\mathrm{PDR}}=3.5 \mathrm{pc}$. The $\Delta A_{V}$ values for the atomic, dark, and molecular $\mathrm{CO}$ gas are 0.8, 2.2, and 4.7, respectively. The PDR model fit is based primarily on the $\mathrm{CO}$, [C I], and [C II] fluxes, but the [O I] fluxes help drive the fit to high $P_{\text {th }}$ and $G_{0}$. The m/o flux ratios vary from 0.63 for [C I $] 2-1$ to 1.4 for $\mathrm{CO} 7-6$, while the more suspect [OI] lines have $\mathrm{m} / \mathrm{o}=$ 3.7 and 0.64 for 63 and $145 \mu \mathrm{m}$, respectively. Since the $63 \mu \mathrm{m}$ line can be self-absorbed, as discussed, we discount the mismatch to the $63 \mu \mathrm{m}$ line. We cannot get higher values for the $145 \mu \mathrm{m}$ line without ruining the fits to the $\mathrm{CO}$ and [C I] lines. Essentially, all of the $[\mathrm{C} \mathrm{II}]$ and IR emission arises from the PDR. This blueshifted gas is likely on the near side of the H II region, moving toward us. We note that this velocity component dominates the line intensity integrated over all velocity and that this position is close to that modeled by Kramer et al. (2008). Our model result for $G_{0}$ is in exact agreement with Kramer et al., but our density at $A_{V}=1$ is $6 \times 10^{4} \mathrm{~cm}^{-3}$, whereas they obtained $2 \times 10^{5} \mathrm{~cm}^{-3}$ in their constant density clumpy model.

(e) The Oberst et al. Car I position $(l, b)=287.370$, -0.630 . The spectra suggest two velocity components. The 


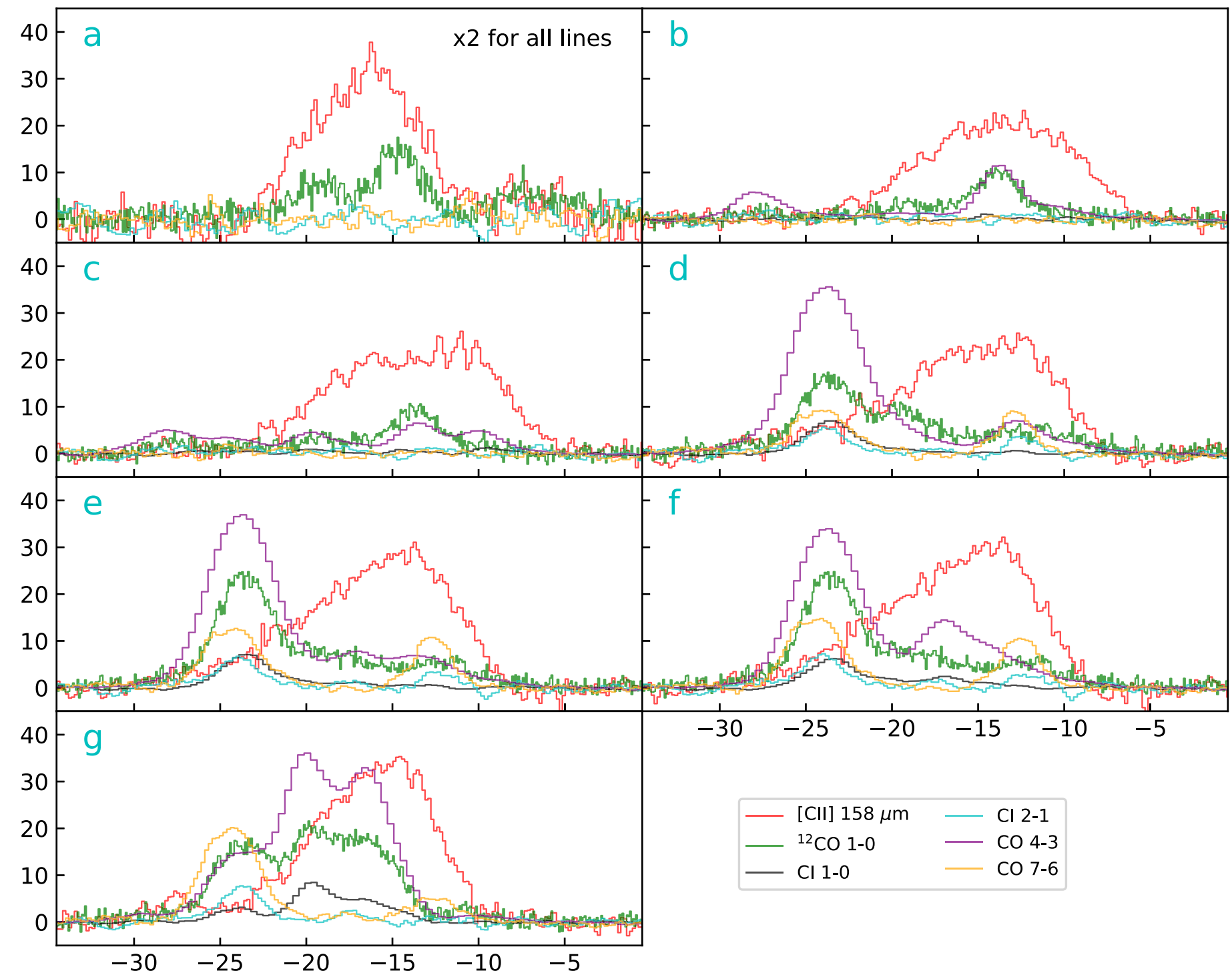

Figure 5. Spectra continued from Figure 4. Spectra of [C II] $158 \mu \mathrm{m}$, [C I] $3 P_{1}-3 P_{0}$ and $3 P_{2}-3 P_{1}$ (Kramer et al. 2008), and CO 4-3 and 7-6 (Kramer et al. 2008) toward seven selected positions for PDR modeling in the Tr $14 /$ Carina I region. The $x$-axis is the LSR velocity in $\mathrm{km} \mathrm{s}^{-1}$. The $y$-axis is the main beam temperature in Kelvin.

first extends from -20 to $-7 \mathrm{~km} \mathrm{~s}^{-1}$. The PDR model fit is $\log P_{\text {th }}=6.5, \log G_{0}=2.5$, and $f_{\mathrm{B}} \simeq 0.5$. The $\Delta A_{V}$ values for the atomic, dark, and molecular $\mathrm{CO}$ gas are $0.2,2.1$, and 2.4 , respectively. The worst $\mathrm{m} / \mathrm{o}=2.0$ for the $[\mathrm{C} \mathrm{I}] 2-1$ line. The other four lines have $\mathrm{m} / \mathrm{o}$ values from 0.7 to 1.5 . The ionized gas is associated with this component, and the [C II] in this velocity range must come from the ionized gas, as the PDR component only contributes roughly $5 \%$. The scaled [N II] lines support this, if we use the electron density estimated from the thermal pressure. Likewise, the $[\mathrm{C} \mathrm{II}] / \mathrm{CO} 1-0$ flux ratio is high, $1.7 \times 10^{4}$, suggesting [C II] from the $\mathrm{HII}$ region. Similarly, this PDR component only supplies about $6 \%$ of the IR continuum. Taking extinction in the $\mathrm{H}$ II region into account, the distance to the PDR is $d_{\mathrm{PDR}}=3.4 \mathrm{pc}$.

The second velocity component extends from -35 to $-20 \mathrm{~km} \mathrm{~s}^{-1}$. The PDR model fit is $\log P_{\mathrm{th}}=6.75, \log G_{0}=$ 3.25 , and $f_{\mathrm{B}} \simeq 0.8$ based on $\mathrm{CO},[\mathrm{CI}],[\mathrm{CII}],[\mathrm{OI}]$, and $\mathrm{IR}$ continuum observations. The $\Delta A_{V}$ values for the atomic, dark, and molecular $\mathrm{CO}$ gas are $0.8,2.2$, and 4.3 , respectively. The fits to the $\mathrm{CO}$ and $[\mathrm{C} \mathrm{I}]$ lines have $\mathrm{m} / \mathrm{o}$ values that range from
1.0 for $\mathrm{CO} 1-0$ and $4-3$ and $[\mathrm{C} \mathrm{I}] 2-1$ to 1.4 for $\mathrm{CO} 7-6$. As in case (d) above, our fit to the [O I] lines matches the $145 \mu \mathrm{m}$ line satisfactorily $(\mathrm{m} / \mathrm{o}=0.7)$, but the model overestimates the $63 \mu \mathrm{m}$ line by a factor of 2.5 . The latter misfit may be caused by self-absorption. ${ }^{23}$ The PDR model fit provides $\sim 90 \%$ of the [C II] emission, so little arises from the ionized gas in this case. This PDR velocity component supplies roughly 0.5 of the observed IR emission. Taking into account moderate extinction in the $\mathrm{H}$ II region, we find $d_{\mathrm{PDR}} \simeq 3.4 \mathrm{pc}$. This blueshifted gas likely lies in the foreground, between the observer and the H II region.

(f) South shell of Carina I-E $(l, b)=287.370,-0.636$. The first velocity component extends from -20 to $-8 \mathrm{~km} \mathrm{~s}^{-1}$. The PDR model fit is $\log P_{\text {th }}=6.75, \log G_{0}=3.0$, and $f_{\mathrm{B}} \simeq 0.5$, based primarily on fits to the $\mathrm{CO}$ and $[\mathrm{CI}]$ lines but also considering the [O I] lines (see below). The $\Delta A_{V}$ values for the atomic, dark, and molecular $\mathrm{CO}$ gas are $0.5,2.2$, and 3.1 , respectively. The $\mathrm{m} / \mathrm{o}$

\footnotetext{
$\overline{23}$ This feature and the second velocity feature in case (d) are the only ones where we could not match the [O I] lines to better than a factor of 2 .
} 
ranges from 1.0 for $\mathrm{CO} 1-0$ and $4-3$ to 1.8 for [C I] $2-1$. The observed [N II] $205 \mu \mathrm{m}$ scales to predict [C II] from the $\mathrm{H}$ II region that is $70 \%$ of the observed. The PDR model only supplies about $10 \%$ of the observed emission. Therefore, the $\mathrm{HII}$ region dominates the [C II] production. This PDR component supplies only $17 \%$ of the observed IR continuum. We show below that the other PDR velocity component contributes about $31 \%$; thus, the $\mathrm{H}$ II region contributes about $52 \%$ of the IR continuum. We find then that the first velocity component lies roughly $d_{\mathrm{PDR}}=4.6 \mathrm{pc}$ from $\operatorname{Tr} 14$. The PDR gas may be somewhat redshifted with respect to the H II gas and therefore may lie somewhat behind UV source $\operatorname{Tr} 14$.

The second velocity component extends from -35 to $-20 \mathrm{~km} \mathrm{~s}^{-1}$. The PDR model fit is $\log P_{\mathrm{th}}=6.5, \log G_{0}=$ 3.0 , and $f_{\mathrm{B}} \simeq 0.9$, based on $\mathrm{CO},[\mathrm{CI}],[\mathrm{C} \mathrm{II}]$, and, to some extent, [O I] and IR continuum. The $\Delta A_{V}$ values for the atomic, dark, and molecular $\mathrm{CO}$ gas are $0.7,2.2$, and 3.4, respectively. The $\mathrm{m} / \mathrm{o}$ for $\mathrm{CO}$ and [C I] ranges from 1.0 for $\mathrm{CO} 1-0$ and 4-3 to 1.4 for $\mathrm{CO} 7-6$. The models predict about equal amounts of [O I] emission from each velocity component. When summed, the $\mathrm{m} / \mathrm{o}=2.7$ for $63 \mu \mathrm{m}$ and 0.85 for $145 \mu \mathrm{m}$. Most of the [C II] in this component comes from the PDR; the PDR model predicts 0.83 of the observed [C II]. In addition, about $31 \%$ of the IR continuum comes from this component. The derived distance to $\operatorname{Tr} 14$ is $d_{\mathrm{PDR}}=4.9 \mathrm{pc}$. The blueshift suggests that this PDR is on the near side of the H II region.

(g) West shell of Carina I-E $(l, b)=287.355,-0.639$. We fit a single velocity component from -30 to $-10 \mathrm{~km} \mathrm{~s}^{-1}$. Here we have no [O I] observations to guide us, but we get an extremely good fit with $\log P_{\text {th }}=6.5, \log G_{0}=2.75$, and $f_{\mathrm{B}} \simeq 1.7$. The $\triangle A_{V}$ values for the atomic, dark, and molecular CO gas are 0.4, 2.1 , and 2.9, respectively. The three CO lines and the two [C I] lines are all fit to within a factor of 1.05 . However, the PDR model fit only provides $28 \%$ of the observed [C II] emission and $31 \%$ of the IR continuum, suggesting that both [C II] and IR arise mostly from the $\mathrm{H}$ II region. Using the inferred extinction in the $\mathrm{H}$ II region, we then estimate $d_{\mathrm{PDR}} \simeq 4.8 \mathrm{pc}$. The blueshifted velocity range suggests that the PDR lies on the near side of the $\mathrm{H}$ II region.

Figure 6 plots pressure $P$ versus $G_{0}$. The asterisks show our PDR code fits of the PDR thermal pressure $P_{\text {th }}$ and $G_{0}$ for 10 spectral features along seven sight lines. The solid line shows the analytic relation of the applied pressure $P_{\mathrm{PDR}}$ to $G_{0}$ for the case where the size scale of the PDR (or clump radius $R$ ) is large compared to the distance to the UV source. The best fits that fall below this line suggest that magnetic pressure helps support the PDR, and that the applied pressure is matched by the sum of the magnetic and thermal pressure. Seven of the 10 fits fall below but within a factor of $\sim 2$ of the applied pressure line. These suggest that the magnetic pressure is only comparable to or smaller than the thermal pressure. Two points fall slightly (much less than a factor of 2) above the relation. Within the errors, these are consistent with thermal pressure dominating. However, one fit (the middle point between $\operatorname{Tr} 14$ and the dust lane) lies a factor of 6 below the applied pressure line. This extreme case may indicate errors in observation (the observations indicate an extremely low CO 7-6/4-3 ratio of $\sim 0.1$, much lower than the other nine observations). However, taking the observations and model fit at face value, it implies a magnetic pressure five times the thermal pressure.

The dotted line at $G_{0}=3000$ indicates how much the applied pressure would rise if the PDR were a clump with radius

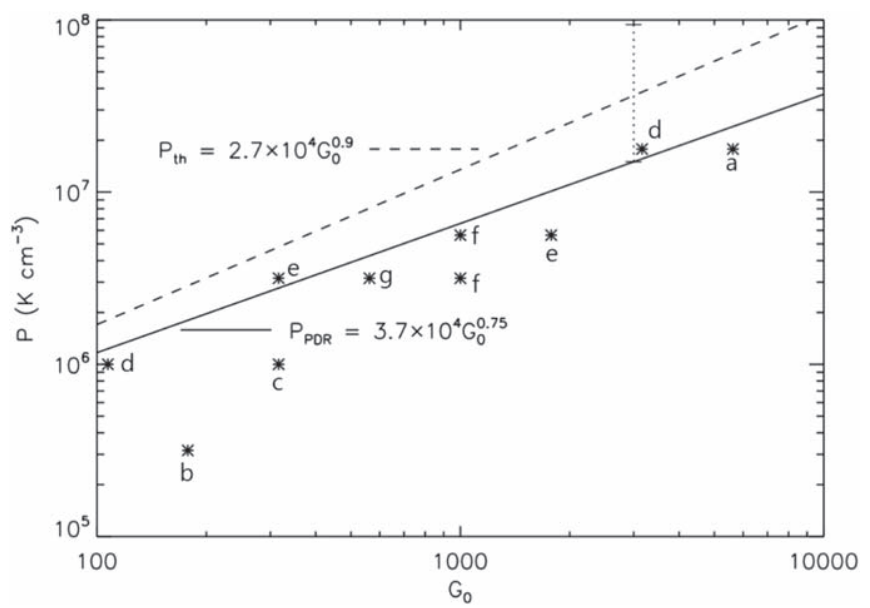

Figure 6. Model thermal pressure in the PDR layer, $P_{\text {th }}$, vs. model incident radiation field $G_{0}$, indicated by the asterisks for the 10 features modeled. The letters denote the seven LOSs. The solid line is our analytic relation, which relates the applied pressure to the PDR, $P_{\mathrm{PDR}}$, to $G_{0}$ for a PDR shell lying just outside the $\mathrm{H}$ II region (Equation (7)). Results that fall below this line might suggest magnetic pressures that support the PDR, rather than thermal pressure. The dashed line is the relation of PDR thermal pressure to $G_{0}$ from Wu et al. (2018). The dotted line shows how much the predicted applied pressure could be raised if the PDR were a $0.1 \mathrm{pc}$ radius clump inside the $\mathrm{H}$ II region.

$R=0.1 \mathrm{pc}$ that is much smaller than the distance to the source ( $d \sim 5$ pc for this FUV field). There are two motivations for showing this result. The first is that the dashed line shows the relation of $P_{\mathrm{th}}$ to $G_{0}$ found by the PDR modeling of Wu et al. (2018). Their fits fall factors of 2-4 above the analytic relation for a shell or clumps with a size larger than $d$. One explanation is that they more often see small clumps in their observations than we do along our LOS. The dotted line shows that clumps of size $\sim 0.1 \mathrm{pc}$ would raise the applied PDR pressure by a factor of about 6 for a given $G_{0}=3000$. The other motivation is that we have one LOS (a, north of $\operatorname{Tr} 14$ ) that is the only one in our analysis that appears to be a clump with a size smaller than $d$. As noted above, its beam filling factor suggests a clump of radius $R \simeq 0.1 \mathrm{pc}$. If this is the case, note that our fit falls below the predicted applied pressure on this clump. This suggests magnetic support for this clump. Indeed, one can compute that if this clump were solely thermally supported, it would gravitationally collapse in a time much shorter than the $\sim 1$ Myr life of the H II region/PDR complex. Therefore, it must be dominated by magnetic pressures.

We conclude this subsection by summarizing the main points of the PDR modeling. The PDRs are observed at both blueshifts and redshifts from the H II region gas, suggesting both foreground and background PDRs. The PDRs on the molecular ridge may have a more edge-on geometry. The distances of the PDRs from the $\mathrm{H}$ II region generally are somewhat greater than $2.3 \mathrm{pc}$, which is the projected distance of the molecular ridge from the $\operatorname{Tr} 14 \mathrm{OB}$ association. This seems reasonable, given the three-dimensional aspect of this blister $\mathrm{H}$ II region and the neutral gas that surrounds it.

Of 10 spectral features analyzed along seven LOSs, we find six spectral features in which the [C II] and IR continuum mainly comes from the $\mathrm{H}$ II region and four where both arise mostly from the PDR. If unresolved spectra had been used, so that we could not separate features along an LOS, we find that out of the seven LOSs, three would have [C II] dominated by PDR emission, two by $\mathrm{H}$ II emission, and two with comparable contributions. As would be expected, the PDRs dominate when the LOSs are directed at the molecular ridge. It is noteworthy 
that [C II] and IR correlate in this way: if one comes primarily from the $\mathrm{H}$ II region, then so does the other. Intuitively, this makes sense. High dust extinction in the $\mathrm{H}$ II region leads to much lower FUV fields incident on the PDR, and thus less [C II] emission. Like other authors (e.g., Oberst et al. 2011), we find that $[\mathrm{CII}]$ often has a significant contribution from the ionized gas in this region around $\operatorname{Tr} 14$. Summing the [C II] luminosities over the seven LOSs, we find that 3.7 times more [C II] luminosity comes from the H II region than from the PDR. The edge-on geometry of the molecular cloud with respect to $\operatorname{Tr} 14$ and the blister geometry leading to expanding H II gas toward and away from us may help explain the importance of the H II region. The high EUV luminosity of $\mathrm{Tr}$ 14 leads to larger columns in the H II gas and therefore stronger [C II] emission from the ionized gas; it also contributes to the possibility of significant dust extinction in the $\mathrm{H}$ II region and therefore dominant contributions to the IR continuum.

The PDR model fits provide a measure of the mass in atomic gas, dark gas $\left(\mathrm{H}_{2}\right.$ but little $\left.\mathrm{CO}\right)$, and $\mathrm{CO}$ gas. Summing the 10 PDRs found on these seven sight lines, we find a beamaveraged mass proportion going as 1:4.1:5.6 for atomic:dark: CO. If these 10 regions are representative, this gives an estimate of the mass budget in this $\operatorname{Tr} 14$ region.

The correlation of the thermal pressure in the PDR with the incident FUV field, observed by $\mathrm{Wu}$ et al. (2018) and confirmed by our PDR models presented here, finds some theoretical basis using a simple analytic model of evaporating ionizing gas pressurizing the PDR surface of a molecular cloud or clump. The good correlation of observation and theory suggests that our PDR models are reasonably correct.

\section{Discussion}

\subsection{Three-dimensional Morphology of the Tr 14/Carina I Region}

We show a schematic picture of the $\operatorname{Tr} 14 /$ Carina I region in Figure 7 illustrating the following six findings about the threedimensional morphology of the Tr 14/Carina I region.

1. The H II region in $\operatorname{Tr} 14 /$ Carina I is expanding and likely to be asymmetric where the redshifted portion of the $\mathrm{H}$ II region is confined by the H I cloud, while the blueshifted portion of the H II region is freely expanding toward us. We find that the $\mathrm{H} 92 \alpha, \mathrm{H} \alpha$, and [N II] $6548 \AA$ lines near Tr 14 and $\operatorname{Tr} 16$ typically have highly asymmetric profiles with long tails to negative velocities relative to the $\mathrm{CO}$ clumps around $-20 \mathrm{~km} \mathrm{~s}^{-1}$ (e.g., the spectra toward positions 1, 5, and 6 in Figure 4). The H I emission in velocity space over the entire $\mathrm{CNC}$ is observed mainly at LSR velocities greater than $-20 \mathrm{~km} \mathrm{~s}^{-1}$, while we do not find significant H I emission at LSR velocities lower than $-20 \mathrm{~km} \mathrm{~s}^{-1}$, indicating that the $\mathrm{H}$ I gas impedes the $\mathrm{H}$ II region from expanding away from us.

2. The $\mathrm{CO}$ and $\mathrm{H}$ I clumps partially confine the $\mathrm{H}$ II regions of $\operatorname{Tr} 14$ and $\operatorname{Tr} 16$. The channel maps in Figures 9-12 show that molecular and $\mathrm{H}$ I clouds/clumps partially surround $\operatorname{Tr} 14$ and Tr 16 in PPV space. We see that there are CO clouds/ clumps surrounding the north, west, and south of Tr 14 and the east of $\operatorname{Tr} 16$.

3. The Tr 14 and $\operatorname{Tr} 16$ clusters have made their own H II regions that have partially merged as they expanded. Figure 8 shows the LSR velocities of the red- and blueshifted peaks of $\mathrm{H} \alpha$ lines observed using Gaia (Damiani et al. 2016). The small separation between the red- and blueshifted peaks indicates that there are either $\mathrm{HI}$ or $\mathrm{CO}$ clumps that confine the $\mathrm{H}$ II regions

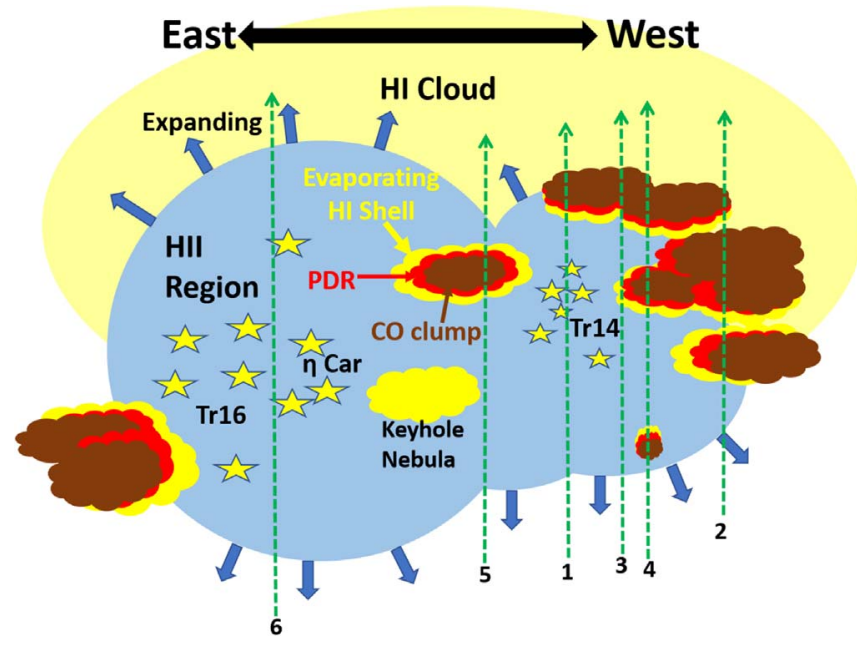

Figure 7. Schematic picture of the CNC. The LOSs toward the six positions are denoted by the green dashed arrows with the position numbers. The green dashed arrows indicate the LOS directions from the observer.

or the edge of the $\mathrm{H}$ II bubble. We find that the separation is reduced between $\operatorname{Tr} 14$ and $\operatorname{Tr} 16$. In the H I $21 \mathrm{~cm}$ map, we see that there are numerous $\mathrm{H}$ I clumps between $\operatorname{Tr} 14$ and $\operatorname{Tr} 16$ separating the $\mathrm{H}$ II regions of the two clusters.

4. The H II region of $\operatorname{Tr} 16$ is likely more extensive than that of $\operatorname{Tr} 14$. A large separation between the red- and blueshifted peaks of the $\mathrm{H} \alpha$ lines (Figure 8) indicates a faster expansion of the $\mathrm{H}$ II region in $\operatorname{Tr} 16$ compared to $\operatorname{Tr} 14$. The expansion speed increases with the radial distance from the ionizing source (e.g., Krumholz et al. 2007); thus, a larger H II region is expected to have a faster expansion speed. We found that $\operatorname{Tr} 16$ has a roughly three times larger projected area and a factor of 2 larger velocity separation between two intensity peaks compared to $\mathrm{Tr}$ 14, suggesting that the $\mathrm{H}$ II region of $\operatorname{Tr} 16$ is significantly larger than Tr 14 along the LOS as well. In addition, CO clouds still confine $\operatorname{Tr} 14$, while $\operatorname{Tr} 16$ seems to have cleared out the nearby dense structures, except the east side.

5. The CO clouds/clumps have PDRs that show a hint of cloud dispersal by photoevaporation and radiation stripping. We find that most of the [C II] emission is spatially correlated with the $\mathrm{CO}$ clouds/clumps, but the intensity peaks of the [C II] emission are slightly displaced from the $\mathrm{CO}$ intensity peaks. In the channel maps, we also found that the morphology of the [C II] emission often has arc-shaped clumps surrounding or a strip adjacent to a $\mathrm{CO}$ cloud, suggesting that there are PDRs and ionization fronts on many of the $\mathrm{CO}$ clouds/clumps. Toward dense clumps (e.g., Carina I-E), most of the H I spectra show absorption features (e.g., positions 3 and 4 in Figure 4). The H I absorption features either red- or blueshifted relative to ${ }^{12} \mathrm{CO}$ emission are likely due to photoevaporation and radiation stripping of the exposed CO clumps as predicted from theories (e.g., Bertoldi 1989; Bertoldi \& McKee 1990; Lefloch \& Lazareff 1994; Mellema et al. 1998; McLeod et al. 2016). This suggests that dense $\mathrm{CO}$ clumps subjected to strong external radiation show a multilayered structure.

6. Some of the $\mathrm{CO}$ clumps are located within the $\mathrm{HII}$ regions. The locations of the ionized, neutral, and molecular gas along the LOS are not trivial to determine, but we think that the $\mathrm{HI}$ absorption features and the [C II] emission provide information about the locations of $\mathrm{CO}$ clumps relative to the H II regions. Near Tr 14, most of the H I spectra show both redand blueshifted absorption features with respect to $\mathrm{CO}$ clumps 


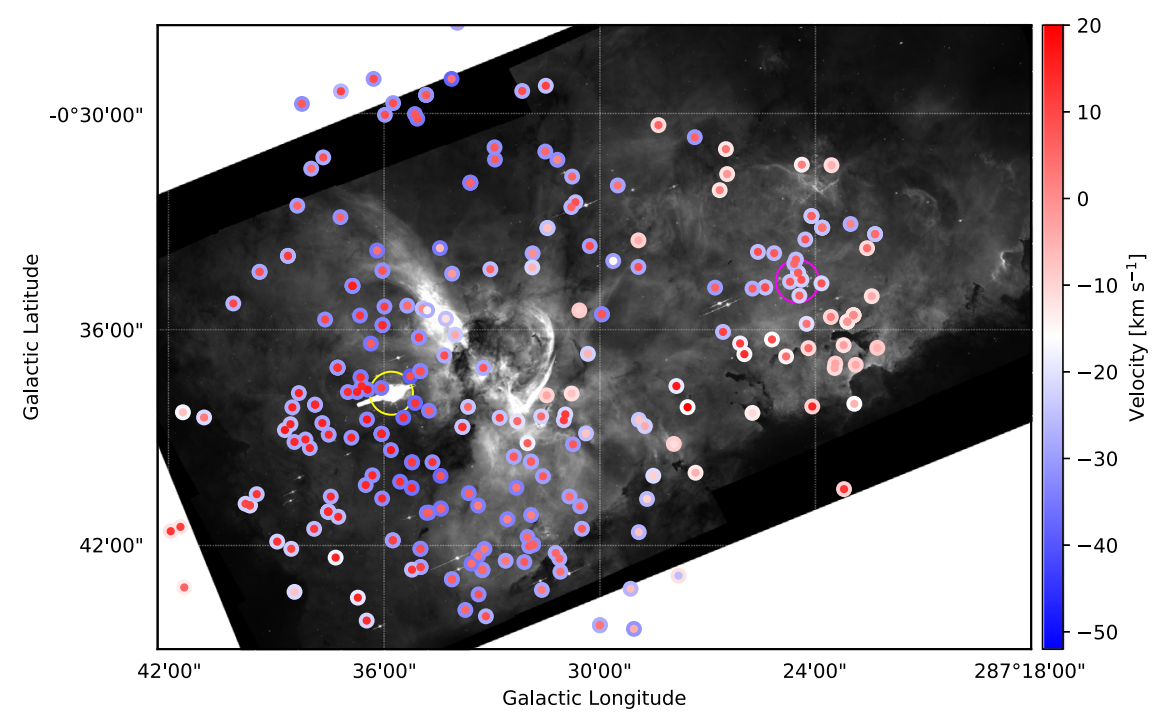

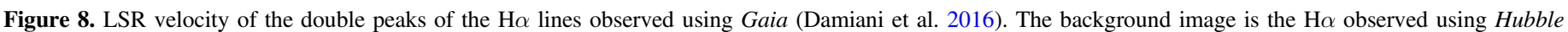

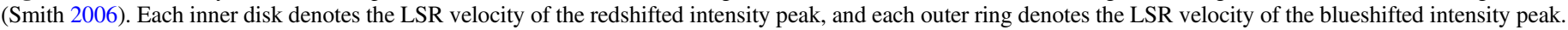
The velocity difference between the red- and blueshifted peaks shows the size of the H II region along the LOS.

at $\sim-20 \mathrm{~km} \mathrm{~s}^{-1}$ (see Figures 9-12). If massive stars powering $\mathrm{H}$ II regions are in front of and behind the $\mathrm{CO}$ clouds/clumps along the LOS, we may observe H I absorption features redand blueshifted relative to the $\mathrm{CO}$ clouds/clumps originating from their evaporating surfaces. The strongest double absorption features are found near Carina I-E and the Keyhole Nebula, suggesting that they are cold dense clumps or pillars located within the $\mathrm{H}$ II regions.

The schematic in Figure 7 presents the six findings on the ISM structure in the $\operatorname{Tr} 14 /$ Carina I region in addition to the descriptions of $\operatorname{Tr} 16$ in Brooks et al. (2000). The H II region is mostly open to us, and the $\mathrm{CO}$ clumps are within the $\mathrm{H}$ II region or partially confine the $\mathrm{H}$ II region. The $\mathrm{CO}$ clumps exposed to the $\mathrm{HII}$ regions have layered structures with evaporating surfaces. There is an $\mathrm{H}$ I cloud enclosing and confining the farside/redshifted portion of the H II region, while the near/ blueshifted side is expanding more freely, similar to the Champagne model (Tenorio-Tagle 1979) but with some of the dense $\mathrm{CO}$ clumps still being left within the $\mathrm{H}$ II regions.

\subsection{EUV Photoevaporation of the GMC}

The GMC, which mostly lies to the west of $\operatorname{Tr} 14$, has an estimated mass of about $\sim 10^{6} M_{\odot}$ (e.g., Grabelsky et al. 1988; Smith \& Brooks 2007). Grabelsky et al. (1988) also estimated the radius of the GMC to be $R_{\mathrm{GMC}} \sim 66 \mathrm{pc}$. Smith (2006) estimated the total present-day EUV photon luminosity produced by the 65 $\mathrm{O}$ stars and the three WNL stars in Carina to be $\Phi_{\mathrm{EUV}} \sim 10^{51}$ EUV photons $s^{-1}$ (see Smith \& Brooks 2007). Of this, about $6 \times 10^{50}$ EUV photons $\mathrm{s}^{-1}$ come from $\operatorname{Tr} 16$, and $2.2 \times 10^{50}$ EUV photons $\mathrm{s}^{-1}$ come from $\operatorname{Tr} 14$. Our STO2 region mostly encompasses the region illuminated by $\operatorname{Tr} 14$, but we examine photoevaporation from our smaller region and also consider the photoevaporation caused by both $\operatorname{Tr} 14$ and $\operatorname{Tr} 16$ as they illuminate the GMC.

We consider a blister configuration for the GMC; that is, $\mathrm{Tr}$ 14 and $\operatorname{Tr} 16$ are assumed at some distance from the surface of the GMC but at a distance that is small compared to the radius of the GMC, $R_{\mathrm{GMC}}$, as well as to the radius $R$ of the illuminated surface. The EUV-induced mass-loss rate from a surface area of radius $R$ is given by

$$
\dot{M}_{\text {evap }} \simeq \pi R^{2} n_{e} \bar{m} v_{\text {evap }}
$$

where $n_{e}$ is the typical electron density at radius $R, \bar{m} \sim 2 \times$ $10^{-24} \mathrm{gm}$ is the gas mass per electron, and $v_{\text {evap }} \sim 10 \mathrm{~km} \mathrm{~s}^{-1}$ is the speed at which the ionized gas moves off the surface of the GMC.

Using Equation (2), we derive

$$
n_{e}(R) \simeq\left(\frac{3 \Phi_{\mathrm{EUV}}}{4 \pi \alpha_{r} R^{3}}\right)^{1 / 2} \simeq 30 \Phi_{51}^{1 / 2} R_{20}^{-3 / 2} \mathrm{~cm}^{-3}
$$

where $R_{20} \equiv R / 10^{20} \mathrm{~cm}$ and $\Phi_{51} \equiv \Phi_{\mathrm{EUV}} / 10^{51} \mathrm{EUV}_{\text {photons s}}{ }^{-1}$. Note that $R$ here is really the distance from the ionizing source to the GMC cloud surface. This is somewhat larger than the radius $R$ of the illuminated surface. However, because we assume that the distance of the stars from the cloud surface is small compared to $R$, these two distances are approximately the same.

Substituting Equation (11) into Equation (10), we obtain

$$
\dot{M}_{\text {evap }} \simeq 3 \times 10^{-2} R_{20}^{1 / 2} \Phi_{51}^{1 / 2} M_{\odot} \mathrm{yr}^{-1} .
$$

Note that although the GMC surface very close $(R \sim 3 \mathrm{pc})$ to $\operatorname{Tr} 14$ and $\operatorname{Tr} 16$ is illuminated with a higher EUV flux and produces a higher mass flux loss, the more distant $(R \sim 30 \mathrm{pc})$ regions of the GMC actually dominate the evaporative mass loss from the cloud because they have so much more area.

The existence of a large area of the cloud being illuminated and ionized by the massive stars in $\operatorname{Tr} 14$ and $\operatorname{Tr} 16$ has been observed by numerous investigators. Mizutani et al. (2004) detected fine-structure IR lines of [O III], [N III], and [N II] and inferred the existence of diffuse $\left(n_{e}<100 \mathrm{~cm}^{-3}\right)$ ionized gas over at least $30 \mathrm{pc}$. Considering just the region dominated by $\mathrm{Tr}$ 14, we see from Equation (11) that although the electron density at the GMC surface at $R \simeq 3 \mathrm{pc}$ is $n_{e} \simeq 450 \mathrm{~cm}^{-3}$, the electron density on the GMC surface at $R \simeq 15 \mathrm{pc}$ is $n_{e} \simeq 40 \mathrm{~cm}^{-3}$, in accordance with the results of Mizutani et al. (2002).

If we substitute $\Phi_{\mathrm{EUV}} \sim 10^{51} \mathrm{~s}^{-1}$ for the total of $\operatorname{Tr} 14$ and $\operatorname{Tr}$ 16 and estimate that their EUV flux covers $R \sim 10^{20} \mathrm{~cm} \simeq 30 \mathrm{pc}$ 
of the GMC surface, then we obtain $\dot{M}_{\text {evap }} \simeq 3 \times 10^{-2} M_{\odot} \mathrm{yr}^{-1}$. If the GMC mass is $M_{\mathrm{GMC}} \simeq 10^{6} M_{\odot}$, then the lifetime of the cloud is $t_{\mathrm{GMC}} \equiv M_{\mathrm{GMC}} / \dot{M}_{\mathrm{evap}} \simeq 30 \mathrm{Myr}$. This is close to the estimated lifetimes of GMCs. Note, however, that this assumes that the cloud is illuminated by $\Phi_{\mathrm{EUV}} \sim 10^{51} \mathrm{~s}^{-1}$ for the entire $30 \mathrm{Myr}$, which implies that other associations will form to replace $\operatorname{Tr} 14$ and $\operatorname{Tr} 16$, once their massive stars die in $~ 3-5$ Myr. If we just use the $\Phi_{\mathrm{EUV}} \simeq 2.2 \times 10^{50} \mathrm{~s}^{-1}$ of $\operatorname{Tr} 14$ and just consider the region mapped by STO2, which is $R \sim 5 \mathrm{pc}$, then the evaporative mass-loss rate from this region is only $\dot{M}_{\text {evap }} \simeq 5 \times$ $10^{-3} M_{\odot} \mathrm{yr}^{-1}$. We will compare this below with the neutral mass loss caused by the expansion of the $\mathrm{H}$ II region in this region.

\subsection{Mass Loss from the GMC Due to Expulsion of Neutral Clumps or Shells}

The escape speed $v_{\text {esc }}$ of a shell or clump of mass $m$ from a $\mathrm{GMC}$ is given by

$$
\frac{1}{2} m v_{\mathrm{esc}}^{2}=\frac{G m M_{\mathrm{GMC}}}{R_{\mathrm{GMC}}} .
$$

Thus,

$$
v_{\mathrm{esc}} \simeq 16\left(\frac{M_{6}}{R_{20}}\right)^{1 / 2} \mathrm{~km} \mathrm{~s}^{-1},
$$

where $M_{6} \equiv M_{\mathrm{GMC}} / 10^{6} M_{\odot}$. Assuming $M_{6} \simeq 1$ and $R_{20} \simeq 2$ (Grabelsky et al. 1988; Smith \& Brooks 2007), we find $v_{\mathrm{esc}} \simeq$ $11 \mathrm{~km} \mathrm{~s}^{-1}$. Assuming the LOS rest velocity of the GMC is $-17 \mathrm{~km} \mathrm{~s}^{-1}$ (Brooks et al. 2003), we then associate all observed $\mathrm{CO},[\mathrm{C} \mathrm{II}]$, and $\mathrm{HI}$ that is traveling at LOS speeds $v<-23$ or $v>-11 \mathrm{~km} \mathrm{~s}^{-1}$ as being liberated from the gravitational potential of the GMC.

We proceed to make a very rough estimate of the mass of neutral gas that is not bound to the GMC and therefore may be considered to be evaporating off the cloud, perhaps pushed by the expanding $\mathrm{H}$ II gas. First, we estimate the mass in neutral gas that is not traced by $\mathrm{CO}$ but by the [C II] from the PDRs. This includes atomic PDR gas but also gas that is $\mathrm{H}_{2}$ but has little $\mathrm{CO}$ due to photodissociation, sometimes called the hidden molecular gas or the dark gas. We assume that most of the [C II] emission comes from the PDRs and not from the $\mathrm{H}$ II ionized gas, and that the [C II] emission is optically thin. This probably gives a lower limit to the mass, since the PDR models show that the [C II] often has a line optical depth of order unity but only a lower limit by a factor of a few. We also assume that the PDR densities are sufficiently high that the level populations are in LTE and that the temperature of the PDR is $T>92 \mathrm{~K}$ (as the PDR models suggest). If the densities or temperature are lower, then again, our estimate is a lower limit. With these caveats, we then obtain a mass of escaping gas (hydrogen and helium included) that is directly related to the [C II] luminosity from the whole region mapped by STO2:

$$
M=1.2\left(\frac{L_{\left[\mathrm{C}_{\mathrm{II}}\right]}}{1 L_{\odot}}\right) M_{\odot} .
$$

The observed STO2 [C II] luminosity from the LOS velocity range -40 to $-23 \mathrm{~km} \mathrm{~s}^{-1}$ is $L_{\left[\mathrm{C}_{\mathrm{II}}\right]}=583 L_{\odot}$. This then corresponds to $707 M_{\odot}$ of atomic and $\mathrm{H}_{2}$ gas (but no $\mathrm{CO}$ gas). The observed STO2 [C II] luminosity from the LOS velocity range -11 to $0 \mathrm{~km} \mathrm{~s}^{-1}$ is $L_{\left[\mathrm{C}_{\mathrm{II}}\right]}=205 L_{\odot}$. This then corresponds to $246 M_{\odot}$ of atomic and $\mathrm{H}_{2}$ (but no $\mathrm{CO}$ ) gas. So, in sum, we find a lower limit of about $1000 M_{\odot}$ of evaporating or nonbound gas traced by [C II].

We use the ${ }^{13} \mathrm{CO} J=1-0$ luminosity to estimate the mass of the evaporating gas traced by $\mathrm{CO}$. This gas is almost certainly optically thin in this isotopic line, so the mass derives directly from the luminosity in the line, the temperature of the emitting gas, and the assumption that the gas density is above the critical density, which the PDR models confirm:

$$
M=9.6 \times 10^{4}\left(\frac{T}{30 \mathrm{~K}}\right)\left(\frac{L^{13} \mathrm{CO}}{1 L_{\odot}}\right) M_{\odot} .
$$

In the 10 spectral features that we modeled in detail, the average temperature of the gas at $A_{V}=5$, typical of the ${ }^{13} \mathrm{CO}$ gas, was $22 \mathrm{~K}$, with a range of $18-33 \mathrm{~K}$. The observed Mopra ${ }^{13} \mathrm{CO}$ luminosity from the LOS velocity range -40 to $-23 \mathrm{~km} \mathrm{~s}^{-1}$ is $L^{13} \mathrm{CO}=0.0077 L_{\odot}$. These numbers then correspond to roughly $540 M_{\odot}$ of $\mathrm{H}_{2}$ gas associated with this blue $\mathrm{CO}$ component. The observed Mopra ${ }^{13} \mathrm{CO}$ luminosity from the LOS velocity range -11 to $0 \mathrm{~km} \mathrm{~s}^{-1}$ is $L^{13} \mathrm{CO}=0.0022 L_{\odot}$. This then corresponds to roughly $155 M_{\odot}$ of $\mathrm{H}_{2}$ gas associated with the red $\mathrm{CO}$ component. In sum, we find about $700 M_{\odot}$ of evaporating or nonbound gas traced by $\mathrm{CO}$. Adding the neutral gas traced by [C II], we find $\sim 1700 M_{\odot}$ of neutral gas in the STO2 mapped region that is unbound to the Carina GMC and possibly evaporating from it.

This range can be compared to the mass in ionized $\mathrm{H} \mathrm{II}$ gas that is evaporating from the GMC in the region mapped by STO2. In the previous subsection, we derived that the ionized mass-loss rate in the STO2 region is $\dot{M}_{\text {evap }} \simeq 5 \times$ $10^{-3} M_{\odot} \mathrm{yr}^{-1}$. Brooks et al. (2001) and Smith (2006) suggested an age for $\operatorname{Tr} 14$ of about $1 \mathrm{Myr}$. Thus, roughly $5000 M_{\odot}$ of ionized gas has evaporated from the surface of the GMC during this time and would mostly still lie inside the STO2 mapped area. This appears to dominate the neutral evaporation by a factor of about 3. In summary, the mass-loss rates we estimate lead to GMC lifetimes of 20-30 Myr if they persist for the cloud lifetime, a result consistent with observational estimates of GMC lifetimes.

\section{Summary}

In this paper, we have presented a [C II] map of the $\operatorname{Tr} 14$ region in the CNC with an angular resolution of $48^{\prime \prime}$ and a velocity resolution of $0.17 \mathrm{~km} \mathrm{~s}^{-1}$. We investigated the distribution of the ISM phases and the kinematics of the $\mathrm{Tr}$ 14 region by comparing our [C II] spectral map to CO $1-0, \mathrm{H} \mathrm{I}$ $21 \mathrm{~cm}, \mathrm{H} \alpha, \mathrm{H} 92 \alpha$, [N II], [O I], and dust continuum. The main results are as follows.

1. The balloon-borne terahertz observatory STO2 has successfully observed [C II] in the $\operatorname{Tr} 14$ region. The typical rms of the $[\mathrm{C} \mathrm{II}]$ map is $1.3 \mathrm{~K}$ in main beam temperature at spatial and velocity resolutions of $48^{\prime \prime}(0.5 \mathrm{pc}$ at $2.3 \mathrm{kpc})$ and $0.17 \mathrm{~km} \mathrm{~s}^{-1}$.

2. The $[\mathrm{C} \mathrm{II}]$ emission is found in a broad region of the $\operatorname{Tr} 14$ region $(83 \%$ and $53 \%$ of the map area, $0.25 \times 0.28$, found to be above 5 and $10 \mathrm{~K}$, respectively). The brightest [C II] emission is $370 \mathrm{~K} \mathrm{~km} \mathrm{~s}^{-1}$ at Carina I-E. The [C II] emission shows good agreement with $8 \mu \mathrm{m}$ PAH emission and warm dust emission at $160 \mu \mathrm{m}$ rather than colder dust emission at $500 \mu \mathrm{m}$. Bright [C II] emission is mostly related to the $\mathrm{CO}$ clumps, while the [C II] emission near the center of the $\mathrm{Tr} 14$ cluster ( $\mathrm{H}$ II region) is relatively weak. This suggests that strong [C II] emission 
Table 1

Telescope Properties

\begin{tabular}{lc}
\hline \hline Facility & STO2 \\
\hline Primary diameter & $80 \mathrm{~cm}$ \\
Rest frequency & $1900.537 \mathrm{GHz}$ \\
Beam size & $48^{\prime \prime}$ \\
Effective resolution $^{\mathrm{a}}$ & $55^{\prime \prime}$ \\
Pointing accuracy $_{\text {Spectral resolution }}$ & $<15^{\prime \prime}$ \\
Velocity resolution $^{\prime}$ & $1 \mathrm{MHz}$ \\
\hline
\end{tabular}

Note.

${ }^{\text {a }}$ Angular resolution of the regridded map.

Table 2

Model Parameters

\begin{tabular}{lc}
\hline \hline Parameter & Value \\
\hline $\mathrm{C} / \mathrm{n}^{\mathrm{a}}$ & $1.6 \times 10^{-4}$ \\
$\mathrm{O} / \mathrm{n}^{\mathrm{a}}$ & $3.2 \times 10^{-4}$ \\
$\mathrm{Mg} / \mathrm{n}^{\mathrm{a}}$ & $1.1 \times 10^{-6}$ \\
$\mathrm{Si} / \mathrm{n}^{\mathrm{a}}$ & $1.7 \times 10^{-6}$ \\
$\mathrm{Fe} / \mathrm{n}^{\mathrm{a}}$ & $1.7 \times 10^{-7}$ \\
$\mathrm{~S} / \mathrm{n}^{\mathrm{a}}$ & $2.8 \times 10^{-5}$ \\
$\delta v_{\mathrm{D}}{ }^{\mathrm{b}}$ & $1.5 \mathrm{~km} \mathrm{~s}^{-1}$ \\
$\zeta_{\mathrm{CR}}{ }^{\mathrm{c}}$ & $2 \times 10^{-16} \mathrm{~s}^{-1}$ \\
$A_{\mathrm{V}}{ }^{\mathrm{d}}$ & 10 \\
\hline
\end{tabular}

Notes.

${ }^{a}$ Gas-phase abundance per hydrogen nucleus.

b Doppler line width.

${ }^{c}$ Primary cosmic-ray ionization rate per hydrogen (Indriolo et al. 2015; Neufeld \& Wolfire 2017).

d Depth of PDR layer.

typically arises from PDRs and H II regions close to the ionization fronts of nearby molecular clouds.

3. The channel maps of [C II] and $\mathrm{CO} 1-0$ show that most of the $\mathrm{CO}$ emission is confined within a velocity range of -32 to $-5 \mathrm{~km} \mathrm{~s}^{-1}$. The bright [C II] emission is typically correlated with $\mathrm{CO}$ clouds/clumps, but the intensity peaks of the [C II] emission are often displaced from the peak intensity of the $\mathrm{CO} 1-0$ emission and located at the outskirts of the CO clouds/clumps in PPV. This indicates that the bright [C II] emission traces the PDRs and ionization fronts of the molecular clouds/clumps.

4. Absorption of the $\mathrm{HI} 21 \mathrm{~cm}$ emission is in good agreement with the $\mathrm{CO}$ clouds/clumps and the Keyhole Nebula, a CO-dark molecular cloud, in position-position space. In velocity space, we find that the absorption peaks of the H I $21 \mathrm{~cm}$ emission are typically offset by $3-15 \mathrm{~km} \mathrm{~s}^{-1}$ in LSR velocity from the intensity peaks of the CO 1-0 and [C II] emission, which is similar to the speed of the dispersing cloud by photoevaporation and radiation stripping in analytic and numerical studies. The rough estimate of evaporated mass is $5000 M_{\odot}$ in the $\operatorname{Tr} 14$ region.

5. We have modeled 10 PDRs from 10 spectral features along seven LOSs using our PDR code to find good fits to the $\mathrm{CO},[\mathrm{CI}],[\mathrm{OI}]$, and [C II] lines and the IR continuum. These fits provide the thermal pressure $P_{\text {th }}$ and $G_{0}$ for each PDR. In general, these pairs lie along a theoretically predicted relation of $P_{\text {th }} \propto G_{0}^{0.75}$ if thermal pressure dominates in the PDR. The empirical relation of $P_{\text {th }}$ to $G_{0}$ found by the Meudon best-fit PDRs in $\mathrm{Wu}$ et al. (2018), $P_{\mathrm{th}} \propto G_{0}^{0.9}$ lies close to the theoretical relation and our best fits but factors of 2-4 above them. This could be due to different physical conditions in the regions mapped by $\mathrm{Wu}$ et al. (2018; for example, small clumps or localized UV sources) or differences in the PDR codes used to find the best fits. In the 10 regions modeled, four regions had the PDR dominating production of the [C II], whereas six regions had the $\mathrm{HII}$ region dominating [C II] production. Averaging over all 10 regions, we found a mass proportion of 1:4.1:5.6 for the atomic:dark:molecular (CO) gas. The dark gas mass in these regions is comparable to the total mass traced by CO. Similarly, we summed the [C II] emission in the 10 regions modeled to get an average ratio in these regions of 3.7 for the [C II] arising from the $\mathrm{H}$ II region to the [C II] arising from the PDR. This ratio is not representative of the entire GMC, since our 10 regions focus on LOSs that penetrate the large blister $\mathrm{H}$ II region around $\operatorname{Tr} 14$.

6. Finally, by combining the distribution of the [C II], $\mathrm{CO} 1-0$, $\mathrm{H} \alpha 92$, H I $21 \mathrm{~cm}$, and optical recombination line emission in PPV space, we find that the three-dimensional morphology of the CNC is consistent with one side of numerous blister $\mathrm{H}$ II regions expanding freely toward us, with each acting in a manner analogous to a Champagne flow. The far side of the $\mathrm{H}$ II regions seems to be confined by either $\mathrm{CO}$ or $\mathrm{HI}$ clouds, showing significant $\mathrm{HI}$ emission at an LSR velocity larger than $0 \mathrm{~km} \mathrm{~s}^{-1}$. The CO clouds/clumps are found at the boundary of the H II region or embedded within the $\mathrm{H} \mathrm{II}$ region. The $\mathrm{HI}$ absorption is correlated with the [C II] and $\mathrm{CO}$ emission in position-position space but is displaced in velocity space, suggesting that the PDR regions highlighted by the strong [C II] emission have evaporating surface or radiation stripping with a velocity around $3-15 \mathrm{~km} \mathrm{~s}^{-1}$. The lifetimes of the GMCs roughly estimated using the cloud dispersal rate measured in [C II] and CO are 20-30 Myr, depending on the assumption of the optical depth of the lines.

We thank the anonymous referee for providing constructive comments that have improved the contents of this paper. We thank Ronin Wu for sharing PACS observations. STO2 is a multi-institutional effort funded by the National Aeronautics and Space Administration (NASA) through the ROSES-2012 program under grant NNX14AD58G. This research was carried out in part at the Jet Propulsion Laboratory, California Institute of Technology, under a contract with NASA. Y.S. acknowledges support from the NASA postdoctoral program. M.G.W. was supported in part by NSF grant AST-1411827. C.K. was supported through NSF grant AST-1410896. Kristina Davis is currently supported by an NSF Astronomy and Astrophysics Postdoctoral Fellowship under award AST-1801983.

\section{Appendix A Intensity Calibration of STO2 Data Using ISO Observations}

We review a method for calibrating the intensity of the STO2 data. A conventional method of calibrating intensity for submillimeter/millimeter data is to use planets. The limited visibility of planets from the Antarctic during the STO2 flight, coupled with limited broadband instrument stability, made such a calibration approach problematic. Oberst et al. (2011) mapped Tr 14 and the Carina Nebula in $158 \mu \mathrm{m}$ [C II] using $I S O$. We use their data to verify the integrated intensity of our STO2 data toward Tr 14/Carina I.

The [C II] data of Oberst et al. (2011) are in units of W $\mathrm{m}^{-2} \mathrm{sr}^{-1}$, while the STO2 data are in $\mathrm{K} \mathrm{km} \mathrm{s}^{-1}$. We convert the 
Table 3

Integrated Intensities of [C II] at 12 Commonly Observed Points Using STO2 and ISO

\begin{tabular}{|c|c|c|c|c|c|}
\hline Points & $\begin{array}{c}l \\
(\mathrm{deg})\end{array}$ & $\begin{array}{c}b \\
(\mathrm{deg})\end{array}$ & $\begin{array}{c}I_{\mathrm{STO} 2}^{*} \\
\left(10^{-4} \mathrm{erg} \mathrm{s}^{-1} \mathrm{~cm}^{-2} \mathrm{sr}^{-1}\right)\end{array}$ & $\begin{array}{c}I_{I I O}^{*} \\
\left(10^{-4} \mathrm{erg} \mathrm{s}^{-1} \mathrm{~cm}^{-2} \mathrm{sr}^{-1}\right)\end{array}$ & $R_{c}$ \\
\hline 1 & 287.256 & -0.535 & 4.0 & 4.1 & 0.98 \\
\hline 2 & 287.256 & -0.585 & 8.1 & 9.2 & 0.88 \\
\hline 4 & 287.405 & -0.536 & 8.8 & 9.2 & 0.96 \\
\hline 5 & 287.255 & -0.687 & 8.3 & 8.2 & 1.02 \\
\hline 6 & 287.254 & -0.634 & 8.0 & 7.7 & 1.04 \\
\hline 9 & 287.255 & -0.687 & 8.8 & 8.6 & 0.96 \\
\hline 10 & 287.305 & -0.686 & 6.6 & 11.5 & 0.59 \\
\hline 11 & 287.355 & -0.686 & 11.5 & 16.3 & 0.71 \\
\hline 12 & 287.405 & -0.686 & 9.1 & 9.6 & 0.94 \\
\hline
\end{tabular}

STO2 data to intensity in units of $\mathrm{W} \mathrm{m} \mathrm{m}^{-2} \mathrm{sr}^{-1}$. Using the Rayleigh-Jeans law, the integrated intensity of an STO2 spectrum is

$$
I_{\text {int, STO2 }}=\int I d \nu=\int \frac{2 k_{\mathrm{B}}}{\lambda^{2}} T_{A} d \nu,
$$

where $I_{\text {int }}$ is the measured integrated intensity, $T_{A}$ is the antenna temperature, $\nu$ is the frequency, $\lambda$ is the wavelength, and $k_{\mathrm{B}}$ is the Boltzmann constant. We can write Equation (17) in terms of velocity as

$$
I_{\text {int }, \mathrm{STO} 2}=\frac{2 k_{\mathrm{B}}}{\lambda^{3}} \int T_{A} d v,
$$

where $v$ is the velocity. Considering a uniform source that fills only the main beam of the antenna, we get the true integrated intensity, $I_{\mathrm{int}}^{*}$, from $T_{A}=\eta_{\mathrm{MB}} T_{\mathrm{MB}}$,

$$
I_{\mathrm{int}, \mathrm{STO} 2}^{*}=\frac{I_{\mathrm{int}, \mathrm{STO} 2}}{\eta_{\mathrm{MB}}},
$$

where $\eta_{\mathrm{MB}}$ is the main beam efficiency converting $T_{\mathrm{MB}}$ to $T_{A}$. For a symmetric Cassegrain antenna such as that of the STO2, a typical value of $\eta_{\mathrm{MB}}$ is 0.7 .

The integrated intensities in Oberst et al. (2011) are calculated using the equation

$$
I_{\text {int,ISO }}^{*}=\frac{\int F_{\nu} d \nu}{\Omega_{I S O}},
$$

where $F_{\nu}$ is the flux of the $I S O$ spectral line ( $\mathrm{W} \mathrm{m}^{-2}$ ), and $\Omega_{I S O}$ is the solid angle of the ISO beam. We assume here that the $I S O$ beam has a circular Gaussian form and that a spectral line has a Gaussian profile. Then, the solid angle is related to the FWHM of the Gaussian beam, $\theta_{\mathrm{FWHM}}$, by

$$
\Omega=\frac{\pi}{4 \ln 2} \theta_{\mathrm{FWHM}}^{2}
$$

The integrated flux is related to the peak flux, $F_{\nu \text {,peak }}$, and the FWHM of the Gaussian fit to the ISO spectral line, $\Delta \nu$, by

$$
\int F_{\nu} d \nu=\sqrt{\frac{\pi}{4 \ln 2}} F_{\nu, \text { peak }} \Delta \nu .
$$

Using Equations (21) and (22), we can rewrite Equation (20) as

$$
I_{\text {int }, I S O}^{*}=\sqrt{\frac{4 \ln 2}{\pi}} \frac{F_{\nu, \text { peak }}}{\theta_{\mathrm{FWHM}}^{2}} \Delta \nu .
$$

Note that the above equation is not the same as Equation (2) in Oberst et al. (2011), which contains typographical errors: the above equation should replace Equation (2) in Oberst et al. (2011).

We were able to compare 12 points in Table 3 from Oberst et al. (2011) to our STO2 map. For a proper comparison, we convolved the STO2 map to have the same beam size as the ISO beam size (70". 1). We excluded the points that are at the edge of the STO2 map. The Galactic coordinates of 12 points, the intensities, and the intensity ratios are given in Table 1 . The intensity ratio of STO2 compared to ISO is

$$
R_{c}=\frac{I_{\mathrm{int}, \mathrm{STO} 2}^{*}}{I_{\mathrm{int}, I S O}^{*}} .
$$

The intensity ratio should be unity if $I S O$ and STO2 are correctly calibrated. We find that the intensity ratio in Table 1 varies somewhat. The median value of the intensity ratio is 0.97 , and the standard deviation is 0.38. Both ISO and STO2 have uncertainties in the data reduction and calibration. Oberst et al. (2011) wrote that their uncertainty in the calibration is about $20 \%$. There are also calibration uncertainties in STO2. Particularly, some of the STO2 data have quite large fringes (baseline ripple). We found that there is a $>30 \%$ uncertainty in the peak intensity depending on the reduction method. Propagating the uncertainties from both ISO and STO2, the uncertainty of the ratio is $36 \%$, which is similar to the standard deviation of the ratio in Table 3.

From having the $1-\sigma$ uncertainty of $R_{c}$ of ${ }_{-0.1}^{0.2}$, we conclude that the STO2 data corrected by the main beam efficiency are consistent with the ISO intensities. A beam efficiency of 0.7 should be employed for the STO2 data, and no further adjustment is included at this time.

\section{Appendix B Channel Maps}

We show channel maps of [C II] ${ }^{12} \mathrm{CO} 1-0, \mathrm{H} 92 \alpha$, and $\mathrm{H} \mathrm{I}$ $21 \mathrm{~cm}$ from -43.5 to $11.5 \mathrm{~km} \mathrm{~s}^{-1}$ (Figures 9-12). These channel maps show the details of the relative distribution of the different ISM phases in PPV space. 


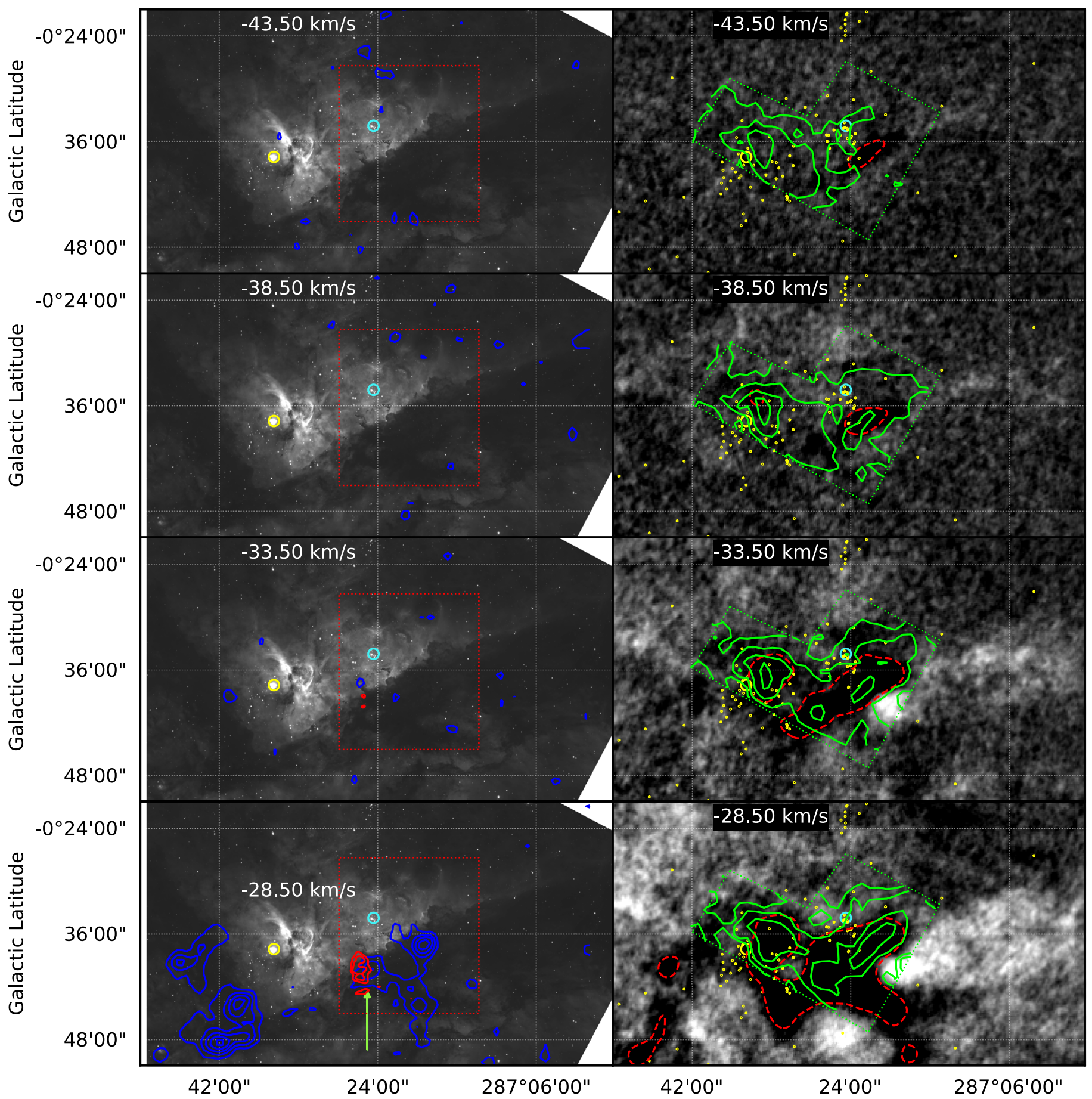

Figure 9. Channel maps of [C II] $158 \mu \mathrm{m}$ obtained with STO2 (red contours) and ${ }^{12} \mathrm{CO}$ observed with Mopra (blue contours; Rebolledo et al. 2016), H92 $\alpha$ (green contours), and H I $21 \mathrm{~cm}$ (gray-scale background in the right column; Rebolledo et al. 2017). The gray-scale background in the left column is an H $\alpha$ image (Smith 2006). The [C II] contours start at $7.5 \mathrm{~K}$ and increase in $2.5 \mathrm{~K}$ increments. The ${ }^{12} \mathrm{CO}$ contours start at $1.5 \mathrm{~K}$ and increase in $1.5 \mathrm{~K}$ increments. The $\mathrm{H} 92 \alpha$ contours are at $0.25,0.5,1,1.5,2$, and $2.5 \mathrm{~K}$. The red and green boxes with the dotted lines are the areas mapped in [C II] and $\mathrm{H} 92 \alpha$, respectively. The red dashed contours are at an antenna temperature of $-30 \mathrm{~K}$ in $\mathrm{H} \mathrm{I} 21 \mathrm{~cm}$ to show the absorption cavity. The large yellow and cyan circles indicate $\eta$ Carinae and the center of the Tr 14 cluster. The yellow dots denote O- and B-type stars (Alexander et al. 2016). The green arrow denotes the [C II] emission associated with a dense CO clump. 


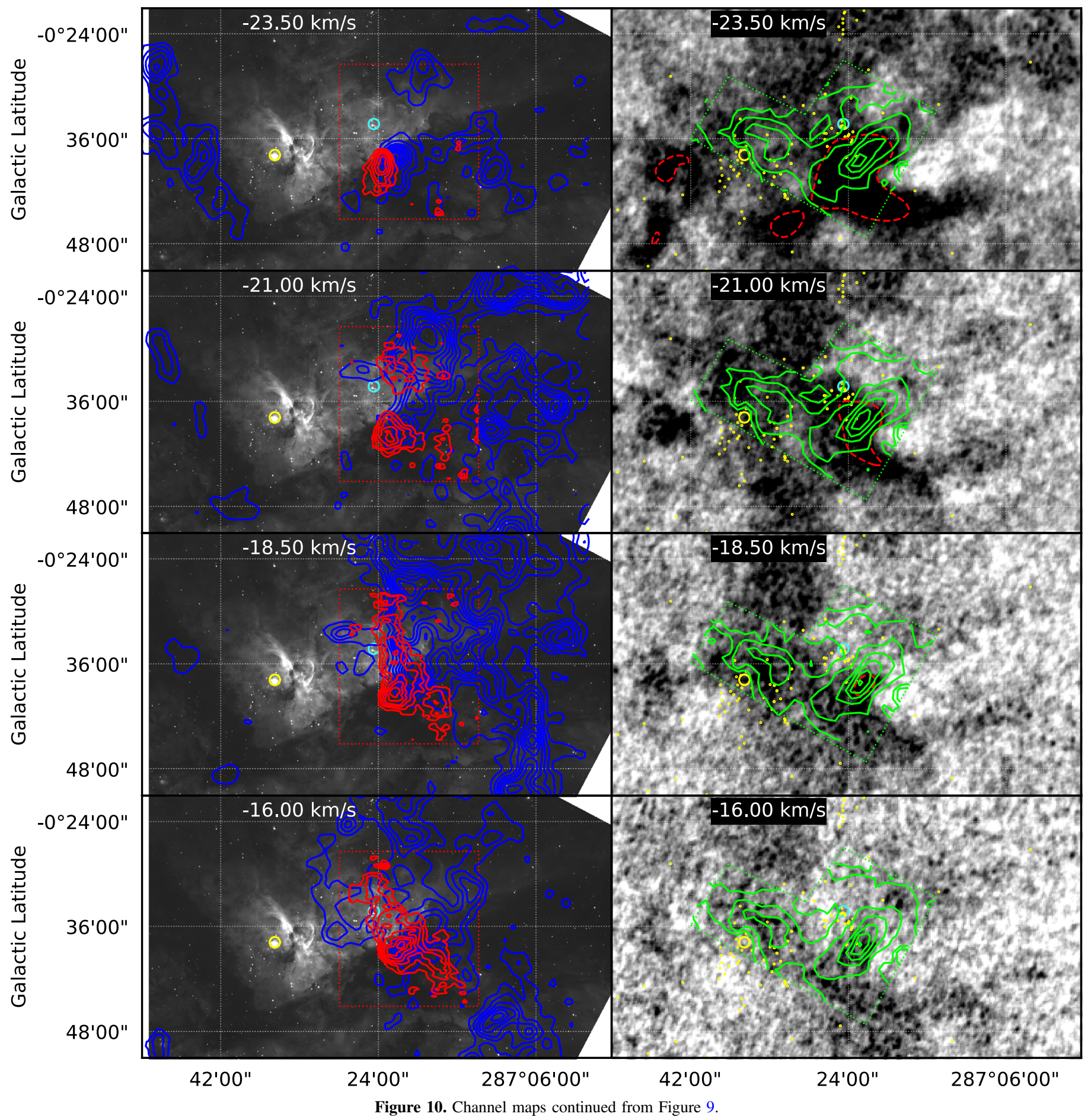




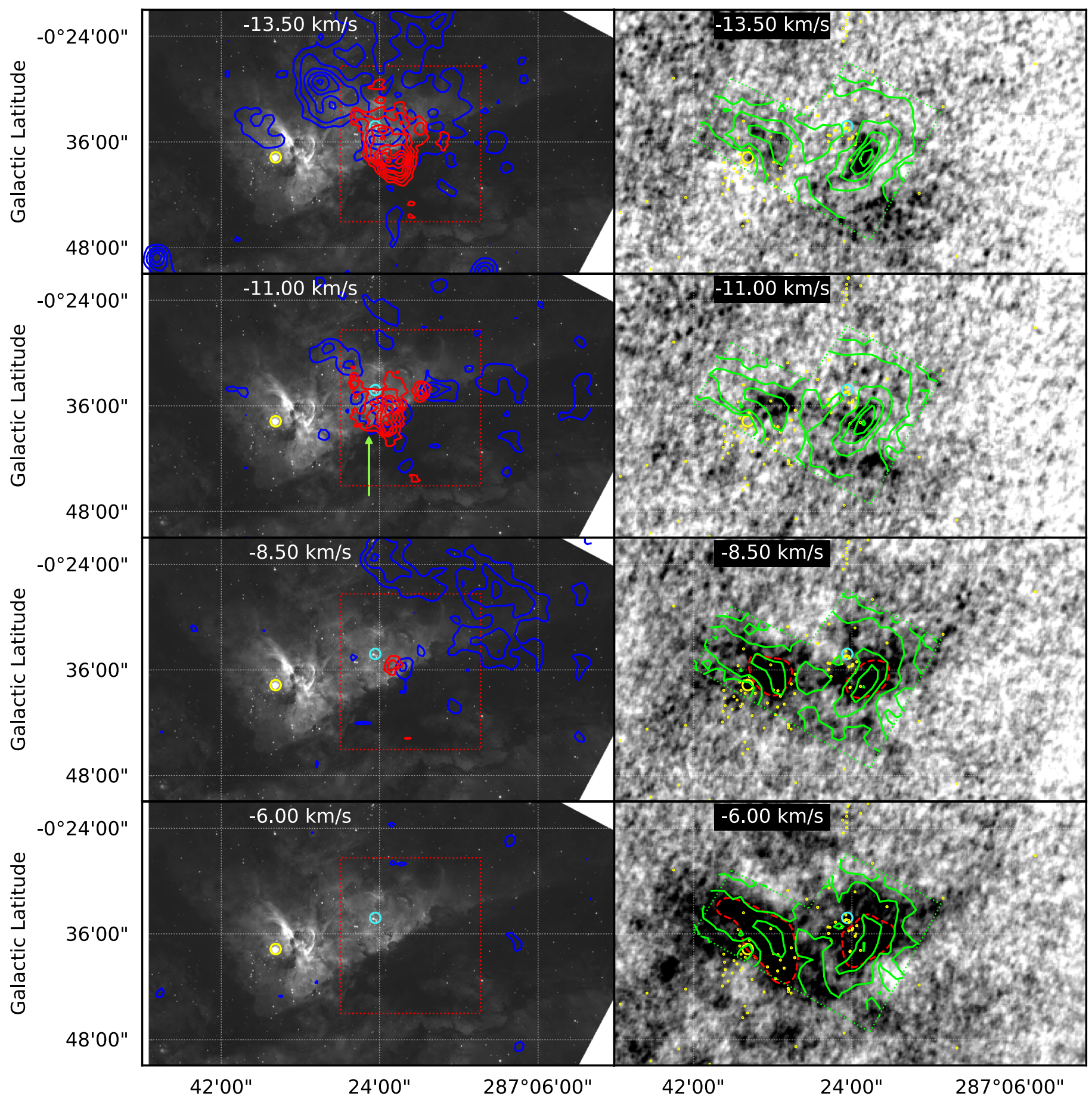

Figure 11. Channel maps continued from Figure 10. 


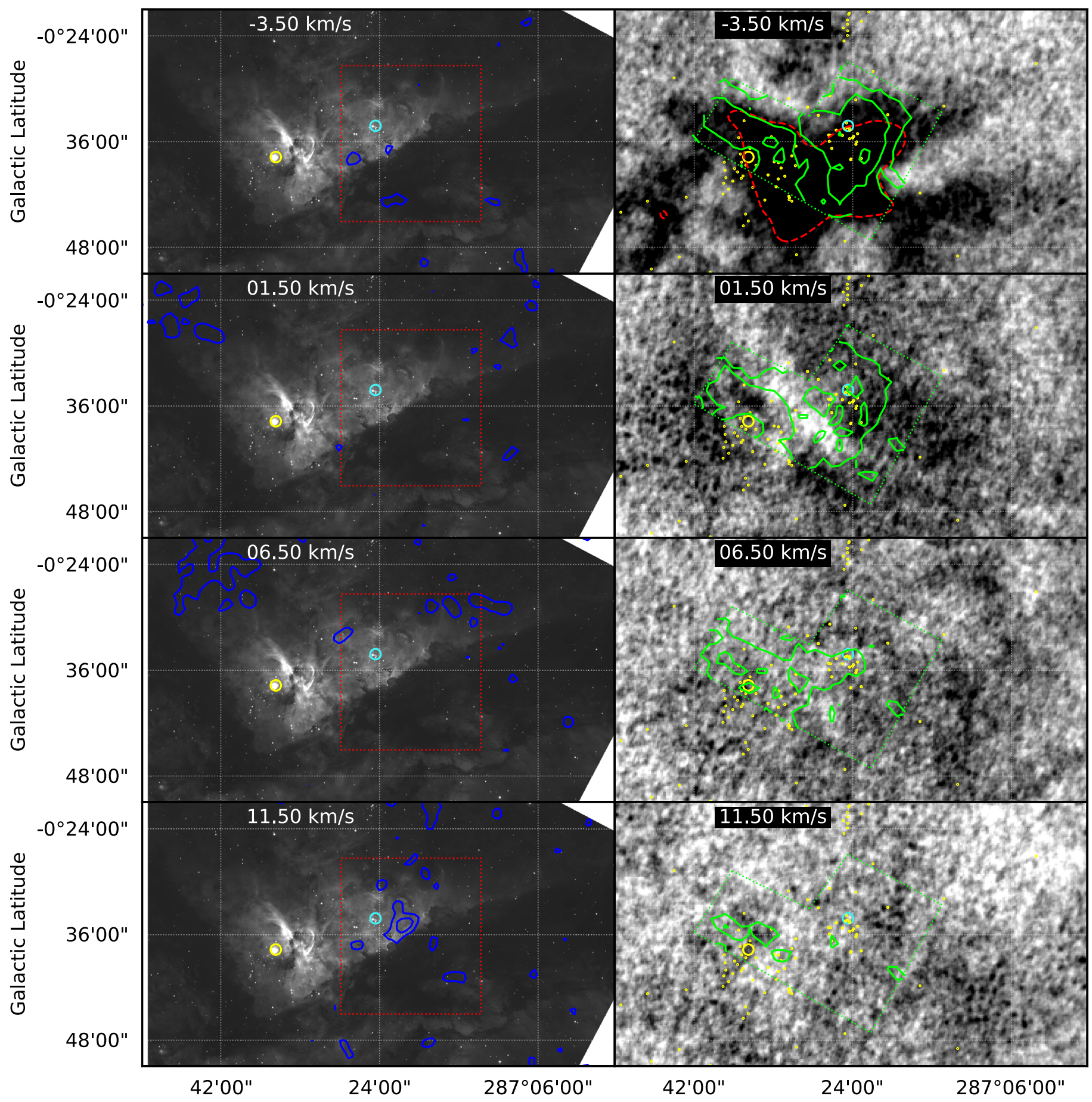

Figure 12. Channel maps continued from Figure 11. 


\section{ORCID iDs}

Young Min Seo (1) https://orcid.org/0000-0003-2122-2617

Paul F. Goldsmith (i) https://orcid.org/0000-0002-6622-8396

Mark G. Wolfire (1) https://orcid.org/0000-0003-0030-9510

Michael G. Burton iㅏ https://orcid.org/0000-0001-7289-1998

Jorge L. Pineda (1) https://orcid.org/0000-0001-8898-2800

Thomas B. Kuiper (1i) https://orcid.org/0000-0003-1798-4918

\section{References}

Alexander, M. J., Hanes, R. J., Povich, M. S., \& McSwain, M. V. 2016, AJ, 152,190

Astraatmadja, T. L., \& Bailer-Jones, C. A. L. 2016, ApJ, 833, 119

Bertoldi, F. 1989, ApJ, 346, 735

Bertoldi, F., \& McKee, C. F. 1990, ApJ, 354, 529

Beuther, H., Ragan, S. E., Ossenkopf, V., et al. 2014, A\&A, 571, A53

Brooks, K. J., Burton, M. G., Rathborne, J. M., Ashley, M. C. B., \& Storey, J. W. V. 2000, MNRAS, 319, 95

Brooks, K. J., Cox, P., Schneider, N., et al. 2003, A\&A, 412, 751

Brooks, K. J., Storey, J. W. V., \& Whiteoak, J. B. 2001, MNRAS, 327, 46

Damiani, F., Bonito, R., Magrini, L., et al. 2016, A\&A, 591, A74

Dias, W. S., Alessi, B. S., Moitinho, A., \& Lépine, J. R. D. 2002, A\&A, 389, 871

Gaczkowski, B., Preibisch, T., Ratzka, T., et al. 2013, A\&A, 549, A67

Gaia Collaboration, Brown, A. G. A., Vallenari, A., et al. 2016a, A\&A, 595, A2

Gaia Collaboration, Prusti, T., de Bruijne, J. H. J., et al. 2016b, A\&A, 595, A1

Gorti, U., \& Hollenbach, D. 2002, ApJ, 573, 215

Grabelsky, D. A., Cohen, R. S., Bronfman, L., \& Thaddeus, P. 1988, ApJ, 331, 181

Haikala, L. K., Gahm, G. F., Grenman, T., Mäkelä, M. M., \& Persson, C. M. 2017, A\&A, 602, A61

Hartigan, P., Reiter, M., Smith, N., \& Bally, J. 2015, AJ, 149, 101

Hollenbach, D., Kaufman, M. J., Neufeld, D., Wolfire, M., \& Goicoechea, J. R. 2012, ApJ, 754, 105

Hollenbach, D. J., \& Tielens, A. G. G. M. 1997, ARA\&A, 35, 179

Hur, H., Park, B.-G., Sung, H., et al. 2015, MNRAS, 446, 3797

Hyvärinen, A., \& Oja, E. 2000, NN, 13, 411

Indriolo, N., Neufeld, D. A., Gerin, M., et al. 2015, ApJ, 800, 40

Kramer, C., Cubick, M., Röllig, M., et al. 2008, A\&A, 477, 547

Krumholz, M. R., Stone, J. M., \& Gardiner, T. A. 2007, ApJ, 671, 518

Langer, W. D., Velusamy, T., Pineda, J. L., Willacy, K., \& Goldsmith, P. F. 2014, A\&A, 561, A122

Le Petit, F., Nehmé, C., Le Bourlot, J., \& Roueff, E. 2006, ApJS, 164, 506 Lefloch, B., \& Lazareff, B. 1994, A\&A, 289, 559

Lindegren, L., Lammers, U., Bastian, U., et al. 2016, A\&A, 595, A4

Malkov, O. Y. 2007, MNRAS, 382, 1073

Mangum, J., Emerson, D., \& Greisen, E. 2000, in ASP Conf. Ser. 217, Imaging at Radio through Submillimeter Wavelengths, ed. J. G. Mangum \& S. J. E. Radford (San Francisco, CA: ASP), 179
McLeod, A. F., Gritschneder, M., Dale, J. E., et al. 2016, MNRAS, 462, 3537 Mellema, G., Raga, A. C., Canto, J., et al. 1998, A\&A, 331, 335

Meyer, D. M., Cardelli, J. A., \& Sofia, U. J. 1997, ApJL, 490, L103

Mizutani, M., Onaka, T., \& Shibai, H. 2002, A\&A, 382, 610

Mizutani, M., Onaka, T., \& Shibai, H. 2004, A\&A, 423, 579

Morrell, N., Garcia, B., \& Levato, H. 1988, PASP, 100, 1431

Neufeld, D. A., \& Wolfire, M. G. 2016, ApJ, 826, 183

Neufeld, D. A., \& Wolfire, M. G. 2017, ApJ, 845, 163

O'Dell, C. R. 2003, The Orion Nebula: Where Stars are Born (Cambridge, MA: Harvard Univ. Press)

Oberst, T. E., Parshley, S. C., Nikola, T., et al. 2011, ApJ, 739, 100

Okada, Y., Pilleri, P., Berné, O., et al. 2013, A\&A, 553, A2

Orr, M. E., Pineda, J. L., \& Goldsmith, P. F. 2014, ApJ, 795, 26

Pabst, C. H. M., Goicoechea, J. R., Teyssier, D., et al. 2017, A\&A, 606, A29

Parravano, A., Hollenbach, D. J., \& McKee, C. F. 2003, ApJ, 584, 797

Pineda, J. L., Langer, W. D., Velusamy, T., \& Goldsmith, P. F. 2013, A\&A, 554, A103

Povich, M. S., Smith, N., Majewski, S. R., et al. 2011, ApJS, 194, 14

Preibisch, T., Roccatagliata, V., Gaczkowski, B., \& Ratzka, T. 2012, A\&A, 541, A132

Rebolledo, D., Burton, M., Green, A., et al. 2016, MNRAS, 456, 2406

Rebolledo, D., Green, A. J., Burton, M., et al. 2017, MNRAS, 472, 1685

Roccatagliata, V., Preibisch, T., Ratzka, T., \& Gaczkowski, B. 2013, A\&A, 554, A6

Rochau, B., Brandner, W., Stolte, A., et al. 2011, MNRAS, 418, 949

Smith, N. 2006, MNRAS, 367, 763

Smith, N., Bally, J., \& Morse, J. A. 2003, ApJL, 587, L105

Smith, N., \& Brooks, K. J. 2007, MNRAS, 379, 1279

Smith, N., \& Brooks, K. J. 2008, in The Carina Nebula: A Laboratory for Feedback and Triggered Star Formation, ed. B. Reipurth (San Francisco, CA: ASP), 138

Smith, N., Povich, M. S., Whitney, B. A., et al. 2010, MNRAS, 406, 952

Smith, N., \& Stassun, K. G. 2017, AJ, 153, 125

Snow, T. P., \& McCall, B. J. 2006, ARA\&A, 44, 367

Sofia, U. J., Lauroesch, J. T., Meyer, D. M., \& Cartledge, S. I. B. 2004, ApJ, 605,272

Szúcs, L., Glover, S. C. O., \& Klessen, R. S. 2014, MNRAS, 445, 4055

Tayal, S. S. 2008, A\&A, 486, 629

Tayal, S. S. 2011, ApJS, 195, 12

Tenorio-Tagle, G. 1979, A\&A, 71, 59

Vazquez, R. A., Baume, G., Feinstein, A., \& Prado, P. 1996, A\&AS, 116, 75

Velusamy, T., Langer, W. D., Goldsmith, P. F., \& Pineda, J. L. 2015, A\&A, 578, A135

Wakelam, V., Bron, E., Cazaux, S., et al. 2017, MolAs, 9, 1

Walborn, N. R. 1973, ApJ, 179, 517

Wolfire, M. G., Hollenbach, D., \& McKee, C. F. 2010, ApJ, 716, 1191

Wolfire, M. G., Hollenbach, D., \& Tielens, A. G. G. M. 1989, ApJ, 344, 770

Wu, R., Bron, E., Onaka, T., et al. 2018, A\&A, 618, 53

Young, T., Horiuchi, S., Green, J. A., \& Jerjen, H. 2013, MNRAS, 435, 3568

Zhang, X., Lee, Y., Bolatto, A., \& Stark, A. A. 2001, ApJ, 553, 274 